\title{
1 Bursting firing in ventromedial hypothalamic neurons exerts dual \\ 2 control of anxiety-like behavior and energy expenditure
}

3

4 Jie Shao ${ }^{1,2,4}$, Dashuang $\mathrm{Gao}^{1,2,4}$, Yunhui $\mathrm{Liu}^{1,2,4}$, Shanping Chen ${ }^{1}$, Nian $\mathrm{Liu}^{1}$, Lu

5 Zhang ${ }^{1}$, Xinyi Zhou ${ }^{1,2}$, Qian Xiao ${ }^{1,2}$, Liping Wang ${ }^{1}$, Hailan $\mathrm{Hu}^{3}$, Fan Yang ${ }^{1,5, *}$

6

7 The Brain Cognition and Brain Disease Institute, Shenzhen Institutes of Advanced

8 Technology, Chinese Academy of Sciences, Shenzhen-Hong Kong Institute of Brain

9 Science-Shenzhen Fundamental Research Institutions, Shenzhen 518055, China.

$10{ }^{2}$ University of Chinese Academy of Sciences, Beijing 100049, China.

$11{ }^{3}$ Department of Psychiatry of First Affiliated Hospital, Zhejiang University School of

12 Medicine, Hangzhou 310058, China.

$13 \quad{ }^{4}$ These authors contributed equally.

$14 \quad{ }^{5}$ Lead contact

$15 *$ Correspondence to: Fan Yang, Shenzhen Institutes of Advanced Technology, Chinese

16 Academy of Sciences, 1068 Xueyuan Boulevard, University Town of Shenzhen, Xili

17 Nanshan, Shenzhen 518055, China. Email: fan.yang@siat.ac.cn; 


\section{Abstract}

Exposure to chronic stress induces anxiety-like behavior and metabolic changes in animals, resulting in adaptive or maladaptive responses to the stressful environment. Recent studies have indicated the dorsomedial ventromedial hypothalamus (dmVMH) as an important hub that regulates both anxiety and energy homeostasis. However, up to now, how dmVMH neurons exert dual control of chronic stress-induced anxiety and energy expenditure remains poorly understood. Here, we established a chronic-stress mouse model that induced anxiety-like behavior, reduced food consumption, and decreased energy expenditure. We found that $c$-fos expression increased and theta band power is higher in the dmVMH after chronic stress, and the proportion of burst firing neurons significantly increased, which was mediated by elevated expression of T-type calcium channel Cav 3.1. Optogenetically evoked burst firing of dmVMH neurons induced anxiety-like behavior, shifted the respiratory exchange ratio toward fat oxidation, and decreased food intake, while knockdown of Cav3.1 in the dmVMH had the opposite effects. Collectively, our study first revealed an important role of $\mathrm{dmVMH}$ burst firing in the dual regulation of anxiety-like behavior and energy expenditure, and identified Cav 3.1 as a crucial regulator of the activity of the burst firing neurons in dmVMH. These molecular and cellular level findings will advance our understanding of the chronic stress-induced emotional malfunction and energy expenditure disorders.

Keywords: Chronic stress; VMH; Burst firing; Anxiety; Energy expenditure 


\section{Introduction}

42 Exposure to stressors regulate neural activity that integrate the aversive behavior and

43 energy expenditure to facilitate stress coping and survival $\frac{1-3}{2}$, however long-term stress

44 causes adverse effects, impairing both mental and physiological functions $4, \underline{5}$ including

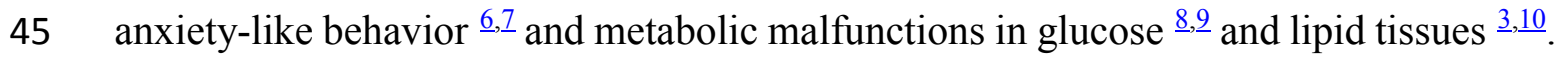

46 Particular effort has been expended to identify the crucial central nodes that

47 interconnect the regulation of emotion and metabolic processes $\underline{3,9,11}$. The hypothalamus,

48 especially the ventromedial hypothalamus (VMH), has been found to play crucial roles

49 in controlling both innate survival behaviors $\underline{12, \underline{13}}$ and energy homeostasis $\underline{11}, \underline{14}, \underline{15}$.

50

51 The $\mathrm{VMH}$ is an evolutionarily conserved deep subcortical nucleus ${ }^{16}$. While the

52 ventrolateral part (vl) of the VMH modulates a series of social behaviors $\frac{17-19}{\text {, the }}$

53 dorsomedial part $(\mathrm{dm})$ is specifically involved in maintaining energy homeostasis

$54 \underline{14}, \underline{15}, \underline{20}, \underline{21}$ and stress response $\mathrm{e}^{13,22}$. Oscillations in the dmVMH can act on sympathetic

55 excitation and regulate energy expenditure $\underline{23,24}$. Steroidogenic factor-1 (SF-1)

56 -expressing neurons are enriched in the dmVMH and play important roles in

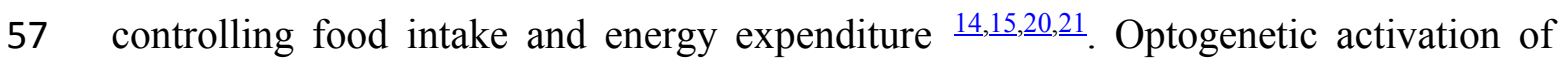

58 VMH SF-1 neurons induces aversive behavior in mice $\stackrel{13,22}{2}$. Furthermore, SF-1 neurons

59 can act as a nutrient-sensitive switch between feeding and anxiety states when facing

60 potential stresses $\stackrel{2}{2}$. Given the complicated functions of SF-1 neurons $\stackrel{19}{\text {, }}$, it is important

61 to dissect how molecular or electrophysiological distinct neuronal subtypes regulate 
62 emotional state and energy homeostasis. However up to now, how dmVMH neurons

63 control neuronal firing to regulate chronic stress-induced anxiety and energy metabolic

64 disorders is elusive.

Recent studies have demonstrated the role of burst firing neurons in regulating emotional state and brain functions; neuronal burst firing and oscillation are critical for the transmission of information and regulation of specific physiological processes in

69 crucial brain areas $\frac{25-30}{}$. In the hippocampus, burst firing of CA1 pyramidal neurons play an important role in regulating N-methyl-d-aspartate (NMDA)-mediated transmission

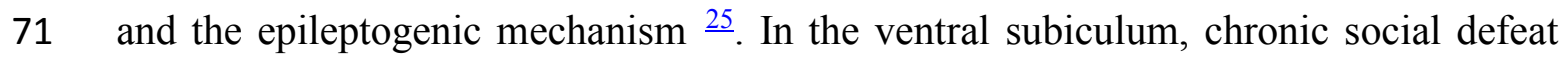

72 stress increases both T-type calcium channel (T-VGCC)-mediated currents and 73 expression of Cav3.1 protein ${ }^{29}$. In the lateral habenula, NMDA-receptor-and- T-VGCC

74 -dependent bursting activity are increased after chronic stress and, most strikingly, can

75 drive depression-like behaviors $\stackrel{26}{2}$. Based on these evidences, we decided to investigate

76 the specific function of bursting firing in the dmVMH, and whether it may be involved

77 in chronic stress-induced anxiety-like behavior and energy metabolic changes.

79 In this study, we first identified that dmVMH neurons can be classified into silent,

80 tonic-firing, and bursting subtypes. We also established a chronic-stress mouse model

81 that induced anxiety-like behavior, reduced food consumption, and decreased energy

82 expenditure. We found a significantly increased proportion of bursting neurons under 
83 chronic stress conditions, which was mainly caused by elevated T-type calcium channel

84 Cav3.1 expression. Importantly, optogenetically-induced dmVMH burst firing drive

85 anxiety-like behaviors, shifted respiratory exchange ratio and decreased average

86 energy expenditure in naïve mice. Conversely, knockdown of Cav3.1 in the dmVMH

87 rescued chronic stress-induced anxiety-like behavior and energy expenditure changes.

88 Taken together, our study first identified Cav3.1-mediated burst firing pattern in the

$89 \mathrm{dmVMH}$, and demonstrated its crucial role in regulating anxiety-like behavior and

90 energy expenditure during the chronic stress.

\section{Results}

93 Chronic stressors induced anxiety-like behavior and energy expenditure change

94 Repeated exposure to stressors modifies the activity of neuronal circuits associated

95 with a variety of abnormalities, including behavior, emotional state, and energy

96 expenditure $\stackrel{2}{ }$, which adaptively or maladaptively respond to the stressful situation. To

97 probe the impact of chronic stress on behavior and energy metabolism, we applied

98 unpredictable chronic stressors to establish a stress mouse model. During the chronic

99 stress period, mice were exposed to random stressors for four weeks, as illustrated in

100 Fig. 1a, with no stressors used in the control group. Both the wild-type and stress

101 groups were tested in a comprehensive phenotyping paradigm. Consistent with

102 previous reports $\stackrel{6}{6}, 7$, we found that chronic stress induced a state of anxiety (Fig. 1b-e).

103 Compared with the control group, mice in the stress group spent less time in the central 
104

105

106

107 and e).

We next explored whether chronic stress affected energy expenditure in stressed mice.

Both the respiratory exchange ratio (RER) and food intake were monitored for $24 \mathrm{~h}$

117 expenditure also showed a significant decrease in stressed mice (Fig. 1h). To determine

118 the underlying cause of different RER in the treatment groups, we also analyzed food

119 intake. As expected, caloric intake in stressed mice was significantly lower than that in

120 the controls, consistent with the known effects of anxiety on food intake (Fig. 1f).

121 Taken together, these findings suggest that chronic stress can induce anxiety-like

122 behavior, reduce food consumption, and shift the RER toward fat oxidation.

124 Given its important role in regulating anxiety, feeding, and energy expenditure $\stackrel{2,21}{2}$, we 
125 hypothesized that the dmVMH may be involved in the above chronic stress-induced

126 changes. After chronic stress, mice were perfused, and c-fos expression was determined

127 via immunostaining. Higher $c$-fos expression was found in the dmVMH of chronically

128 stressed mice than in that of the control group (Fig. 1i), suggesting that more dmVMH

129 neurons were activated under chronic stress conditions. We then employed in vivo

130 electrophysiology to record neuronal activity in the dmVMH. Mice were implanted

131 with $\mathrm{Ni}-\mathrm{Cr}$ electrodes into the dmVMH after chronic stress induction (Fig. 1j), and

$132 \mathrm{dmVMH}$ neuronal activity was monitored after recovery from surgery. The local field

133 potential (LFP) results demonstrated that stressed mice exhibited higher theta band

134 power in the dmVMH than that of control mice (Fig. 1k-1). Together with the $c$-fos

135 staining results, our data suggest that dmVMH neuronal activity is significantly

136 changed after chronic stress.

\section{Chronic stressors induced burst firing in dmVMH}

139 The above experiments revealed enhanced neuronal activity in the dmVMH under

140 chronic stress, but the underlying mechanism was unclear. To explore which

141 electrophysiological characteristics of dmVMH neurons changed after chronic stress,

142 we recorded $84 \mathrm{dmVMH}$ neurons from 38 stressed mice and $85 \mathrm{dmVMH}$ neurons from

14332 control mice under whole-cell patch clamp configuration and analyzed the

144 electrophysiological characteristics. Our data demonstrated that these neurons

145 displayed shorter onset time and depolarized RMP, on average, compared with the 

neurons was enhanced under chronic stress, consistent with the $c$-fos immunostaining

148 and in vivo electrode recording results.

150 We carried out a comprehensive analysis of the membrane properties of dmVMH

151 neurons to quantitatively determine their electrophysiological diversity (Fig. 2b). To

152 classify these neurons, we measured and analyzed eight electrophysiological

153 parameters (Table 1) and performed cluster analysis. The resulting dendrogram in Fig.

$1542 \mathrm{~b}$ (with rescaled distance shown along the vertical axis) illustrates the similarity

155 between clusters. Furthermore, cluster analysis showed that all neurons could be

156 divided into three subtypes: i.e., silent, tonic-firing, bursting. These three dmVMH

157 neuronal subtypes displayed distinct electrophysiological properties (Fig. 2b, Extended

158 Data Fig. 1).

159

160 We then applied cluster analysis using the same methods as depicted above (Fig. 2d) to

161 explore overall changes in the electrophysiological properties of dmVMH neurons after

162 chronic stress. We found that dmVMH neurons in stressed mice could also be divided

163 into three subtypes. Onset time, RMP and recording site of these three subtypes were

164 also compared with those of the control group (Extended Data Fig. 2). By analyzing

165 data from behavior tests and electrophysiological recordings, we found the proportion

166 of burst firing neurons in stressed group, especially in mice with obvious anxiety-like 
167 behavior (anxiety group), increased significantly compared with that in the control

168 group, whereas the proportion of the other subtypes decreased (Fig. 2d-e). This

169 alteration could explain why dmVMH neurons from stressed mice demonstrated a

170 shorter average onset time of action potentials induced by $50 \mathrm{pA}$ current injection (Fig.

171 2a), as burst firing neurons displayed a similar onset time as tonic-firing neurons but a

172 shorter onset time compared with silent neurons (Fig. 2f, Extended Data Fig. 2b). In

173 addition, the increased proportion of bursting neurons and more depolarized

174 tonic-firing neurons (Fig. 2f, Extended Data Fig. 2a) may have contributed to the higher

175 average RMP in dmVMH neurons after chronic stress (Fig. 2a). Furthermore, the

176 average inter-spike interval (ISI, between first and second spike of a burst) of dmVMH

177 burst firing neurons in anxiety group was shorter than that of the control group

178 (unpaired Student's $t$-test, $P=0.0173$ ), and a shorter ISI represents higher frequency

179 firing in a single burst (Fig. 2g). We also found that ISI of dmVMH bursting neurons

180 was more correlated with the time in central area or open arms in mice of

181 stress-induced anxiety compared with the mice in control group (Fig. 2h,i). Taken

182 together, these results demonstrate a possible link between increased bursting activity

183 in the dmVMH and chronic stress induced anxiety.

185 Optogenetic manipulation of burst firing neurons in dmVMH

186 Given the enhancement of burst firing in the dmVMH after chronic stress, we 187 investigated whether induced burst firing of dmVMH neurons alone in unstressed mice 
199 We then applied low-frequency yellow light illumination to induce burst firing in vivo and performed behavioral and energy metabolic tests in NpHR-expressing mice (Fig.

$2013 \mathrm{~d})$. This led to decreased residence time in the central area of the open field and open

202 arm of the elevated plus maze, mimicking the effects of chronic stress-induced

203 anxiety-like behavior (Fig. 3e,f). We also applied wireless optogenetics to induce burst

204 firing of neurons and simultaneously monitored the metabolism of mice using CLAMS.

205 We applied 0.1-Hz yellow light illumination at the start of the test period for $2 \mathrm{~h}$ and 206 repeated the stimulation after $12 \mathrm{~h}$, with consecutive monitoring of mouse energy 207 metabolism for $24 \mathrm{~h}$. Results indicated that average RER, energy expenditure, and food 208 intake in the test period decreased significantly compared with the baseline level before 
209 the test period (Fig. 3g-i). Taken together, our results indicated that enhancement of

210 burst firing induced by local optogenetic manipulation in the dmVMH was sufficient to

211 affect anxiety-like behavior and energy expenditure changes, which simulated the

212 phenotypes of chronically stressed mice.

\section{Burst firing in dmVMH is mediated by T-VGCC}

215 Bursting is an important firing pattern in neural systems and is essential for specific

216 information transmission and function regulation ${ }^{30}$. The T-VGCC, including its three

217 isoforms (Cav3.1, Cav3.2, and Cav3.3), is a pacemaker that can generate LTS $\underline{26}, \underline{31}$.

218 Unlike hyperpolarization-activated cyclic nucleotide-gated channel (HCN), the

219 T-VGCC can induce higher frequency firing and has a threshold near to RMP. It has

220 been reported that Cav 3.1 and Cav 3.2 alone display strong burst firing with a

221 low-voltage threshold, whereas Cav3.3 contributes to burst firing in a different way $\underline{31}$.

222 The expression of T-VGCC is highly correlated with T-type calcium currents, which

223 directly affect the strength and width of bursting $\underline{29}, \underline{32}$. Given the known role of T-VGCC

224 in burst firing, we examined T-VGCC expression in the dmVMH after chronic stress.

226 Based on immunohistochemical analysis, Cav3.1, Cav3.2, and Cav3.3 were all found to

227 be expressed in the dmVMH of naïve mice. The signals of Cav3.1 were much stronger

228 than those of Cav3.2 and Cav3.3 (Fig. 4a). To investigate whether differential

229 expression of the T-VGCC occurs after chronic stress, we performed acute dissection of 
$230 \mathrm{dmVMH}$ tissue from brain slices and harvested single dmVMH cells after patch

231 recordings to extract RNA, then performed qRT-PCR to quantify T-VGCC expression.

232 The expression of Cav3.1 in the dmVMH of stressed mice was significantly higher than

233 that of the control group, whereas no obvious changes were observed in Cav 3.2 or Cav

$234 \quad 3.3$ at either the tissue or single-cell level (Fig. 4b-c).

235

236 To further confirm the contribution of the T-VGCC in dmVMH burst generation, we

237 applied mibefradil, an antagonist of T-VGCC, in whole-cell recording experiments.

238 Results indicated that the burst firing of dmVMH neurons elicited by a 10-pA current

239 injection was indeed inhibited (Fig. 4d). Furthermore, we found that application of

240 mibefradil increased the onset time in the dmVMH neurons of the stress group, but not

241 in the control group, but the change in RMP was similar between the two groups (Fig.

242 4e). Moreover, as we collected single neurons after whole-cell recordings for T-VGCC

243 quantification, we combined the single-cell qRT-PCR and electrophysiological

244 classification data to determine the differential expression of Cav3.1 in the dmVMH

245 neuronal subtypes. Results showed that Cav3.1 expression was much more enriched in

$246 \mathrm{dmVMH}$ bursting neurons, especially after chronic stress (Fig. 4f). Consistently, the

247 Cav3.2 antagonist ascorbate $\underline{33}$ did not significantly affect burst firing of dmVMH

248 neurons (Extended Data Fig. 3). We also investigated the effects of mibefradil on the

249 frequency-current curves using the same stimulus protocol, and found that the firing

250 rate of bursting neurons was decreased by bath application of mibefradil $(P=0.007$, Fig. 
$2514 \mathrm{~g}$, right), but the changes in the firing rate were much more significant in the stress

252 group than in the control group ( $P=0.0047$, Fig. 4h, upper). These data support the idea

253 that the T-VGCC, especially the Cav3.1 isoform, mediates burst firing in the dmVMH.

255 To investigate the in vivo effects of T-VGCC blockade, we bilaterally implanted a 256 cannula and precisely delivered mibefradil or saline into the dmVMH of chronically 257 stressed mice to block burst firing (Extended Data Fig. 4a). Results showed that 258 mibefradil infusion increased the time spent in the central area of the open field and 259 open arm of the elevated plus maze compared with the control group treated with saline 260 (Extended Data Fig. 4b). We also investigated energy metabolic changes after oral 261 administration of mibefradil, and found it to have no effect on food intake. However, 262 administration of mibefradil significantly rescued the decreased RER found in stressed 263 mice. Taken together, these data indicate that the T-VGCC in the dmVMH mediates 264 chronic stress-induced behavioral and energy expenditure changes.

266 The function of the T-VGCC is tightly related to postsynaptic excitatory/inhibitory state

$267 \underline{34,35}$. Several studies have suggested that the generation of burst firing mediated by

268 T-VGCC activation in certain regions is highly dependent on membrane potential $\underline{35,36}$,

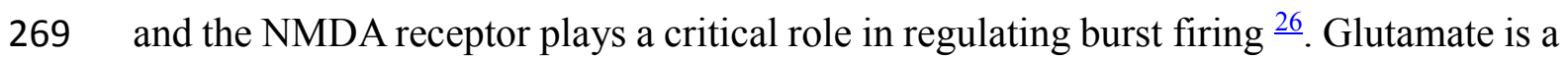

270 common excitatory neurotransmitter and its concentration around neurons may affect

271 membrane potential $\stackrel{37}{ }$. We applied different glutamate receptor antagonists into 
272 artificial cerebrospinal fluid (ACSF) during whole-cell recordings of burst firing

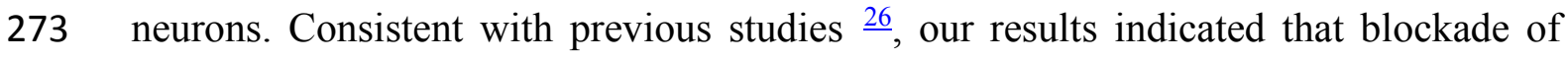

274 NMDA receptors, but not AMPA receptors, significantly inhibited burst firing and

275 caused membrane hyperpolarization, which made it more difficult to reach the

276 T-VGCC threshold (Extended Data Fig. 5).

278 Knockdown of Cav3.1 in dmVMH decreased burst firing and ameliorated chronic

279 stress response

280 Given the important role of Cav 3.1 in mediating burst firing in the dmVMH, we next

281 tested whether down-regulation of Cav 3.1 expression in the dmVMH was sufficient to

282 ameliorate anxiety-like behavior and energy metabolic changes induced by chronic

283 stress. We developed a lentivirus-mediated RNAi method to interfere with expression

284 of Cav3.1 in the dmVMH under chronic stress, with the shRNA-expressing vector

285 injected into the dmVMH bilaterally prior to chronic-stress exposure (Fig. 5a). To test

286 the efficacy of shRNA-mediated Cav3.1 silencing, we used immunofluorescence to

287 confirm the efficient knockdown of Cav3.1 in the dmVMH of stressed mice (Fig. 5b).

288 The effects of Cav3.1 knockdown on burst firing were tested using whole-cell

289 recording on brain slices obtained from Cav 3.1 knockdown mice. Results showed that

2907 out of $17 \mathrm{dmVMH}$ neurons from stressed mice displayed evoked burst firing, whereas

291 only 2 out of 13 dmVMH neurons succeeded after Cav3.1 knockdown (Fig. 5b),

292 suggesting burst firing inhibition induced by RNAi of Cav3.1. 
293 To investigate phenotypic changes in Cav3.1 knockdown mice, we carried out

294 behavioral and energy metabolic tests after viral expression of shRNA of Cav3.1 for

$2954-5$ weeks. Importantly, behavioral tests showed that Cav 3.1 knockdown significantly

296 increased residence time and entries into the central area of the open field and open arm

297 of the elevated plus-maze of stressed mice (Fig. 5c,d), suggesting that local knockdown

298 of Cav 3.1 in the dmVMH was sufficient to rescue stress-induced anxiety-like behavior.

299 For energy metabolic tests, we fasted all mice overnight and analyzed energy

300 expenditure using CLAMS. Data showed that Cav 3.1 silencing effectively rescued the

301 chronic stress-induced decrease in RER (Fig. 5f), as well as the reduction in food intake

302 (Fig. 5f) and energy expenditure (Fig. 5a and h). No obvious body weight differences

303 were observed among the control, stress, and RNAi groups (Fig. 5e).

305 Together with our optogenetic manipulation experiments, our results consistently

306 demonstrated that Cav 3.1 in the dmVMH was both sufficient and necessary to elicit

307 burst firing of dmVMH neurons. Furthermore, knockdown of Cav 3.1 in the dmVMH

308 ameliorated chronic stress-induced phenotypic abnormalities, including anxiety-like

309 behavior, lower RER, and decreased food intake.

\section{Discussion and conclusion}

312 Previous studies have indicated that the dmVMH is an important stress coping center to

313 balance aversive behavior and energy seeking ${ }^{2}$ and may be a possible hub connecting

314 stress-induced emotion and energy expenditure. Here, we reported that chronic stress 
315 induced burst firing enhancement in the dmVMH, which was critical for inducing

316 anxiety-like behavior and energy expenditure alteration in stressed mice. Bursting is an

317 important firing pattern in the central nervous system and is also important for specific

318 information transmission. Based on our data, we determined that dmVMH burst firing

319 neurons play an important role in connecting the emotional state of anxiety and energy

320 homeostasis, and that T-VGCC Cav 3.1 is essential for dmVMH burst firing and

321 contributes to chronic stress-induced behavior and energy expenditure changes (Figure

322 S6).

$\mathrm{dmVMH}$ is responsive in chronic stress

325 Chronic unpredictable stress is detrimental to both mental and physical health.

326 Responses to chronic stress can vary from emotional disorders, like anxiety and

327 depression, in the central nervous system to disturbed metabolism and energy

328 homeostasis in peripheral organs $\stackrel{3-5}{ }$. However, how these physiological processes

329 integrate in the brain and the underlying neural mechanisms remain poorly understood.

330 The dmVMH is implicated in integrating information in the limbic system and in

331 maintaining energy balance $\stackrel{38}{ }$. Furthermore, SF-1 neurons in the dmVMH constitute a

332 nutritionally sensitive switch, which modulates the competing motivations of feeding

333 and avoidance of environments full of acute stress $\stackrel{2}{2}$. Given the important role of the

$334 \mathrm{dmVMH}$ in balancing acute stress inputs and food-seeking, the effects of persistent

335 unpredictable stress on the function of $\mathrm{dmVMH}$ neurons require further research. 
337 In the current study, the dmVMH was found to be involved in integrating chronic stress

338 inputs to regulate anxiety-like behavior and energy expenditure. We established an

339 unpredictable and persistent stress mouse model and characterized the model with

340 multiple energy metabolic, electrophysiological, and behavioral approaches.

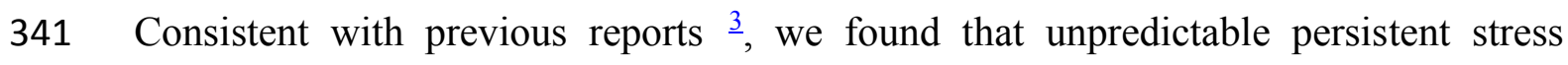

342 induced aversive behavior, decreased food intake, and shifted the RER toward fat

343 oxidation, without obvious effects on body weight. Furthermore, in vivo

344 electrophysiology demonstrated that the power density of the theta band of local field

345 potential was higher in the stress group than in the control group. To dissect the

346 alteration in dmVMH neuronal activity under chronic stress conditions, we studied the

347 electrophysiology of dmVMH neurons. Based on differences in electrical activity, we

348 classified the dmVMH neurons into three subtypes: i.e., silent, tonic-firing, and

349 bursting. By comparing these subtypes in the control and stress groups, we found an

350 enhancement of burst firing in the dmVMH after chronic stress, including increased

351 percentage of burst firing neurons, enhanced LFP, and decreased ISI of bursts. Using

352 patch clamp recordings, our data demonstrated that chronic stressors indeed induced

353 obvious electrophysiological changes in dmVMH neurons at the cellular level, further

354 supporting the important role of the dmVMH in chronic stress. 
357 Action potentials that arrive in bursts provide more precise information than action

358 potentials that arrive singly and further enhance the efficacy of neuromodulator release,

359 thus revealing the special role of bursts in information transmission and processing $\underline{30}$.

360 Many previous studies have indicated that burst firing of specific neuronal

361 subpopulations is critical for performing specific functions. In the hippocampus, a

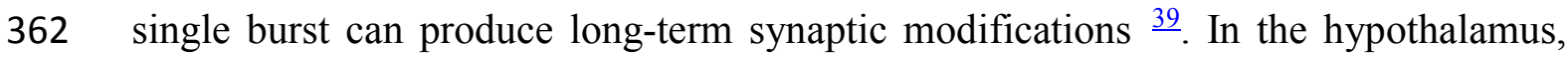

363 burst firing is found in several regions, including the preoptic area and VMH $\stackrel{40}{\text {. In the }}$

364 lateral habenula, bursting activity depends on the NMDA receptor and T-VGCC; and

365 most importantly, can drive behavioral aversion and depression-like symptoms $\underline{26}$. To

366 date, however, no specific study has reported on the functions of dmVMH burst firing

367 under chronic stress. Here, we performed electrophysiological and gene expression

368 studies and demonstrated that burst firing neurons in the dmVMH are actively

369 involved in chronic stress response, and the ISI of burst firing was significantly

370 correlated with the anxiety-like behavior in anxious mice after chronic stress.

372 To mimic burst firing in vivo, we applied optogenetic inhibition with an

373 NpHR-expressing AAV vector, as optogenetic inhibition on the lateral habenula or

374 hippocampal NpHR-expressing neurons can elicit burst firing in yellow light

375 illumination intervals $\stackrel{26,41}{\underline{4}}$. However, burst firing in the dmVMH could not be elicited

376 under an illumination frequency greater than $0.2 \mathrm{~Hz}$ in our electrophysiological

377 studies, indicating regional specificity of T-VGCC activation kinetics. Importantly, we 
378 applied wireless optogenetics to successfully achieve burst manipulation in mice

379 residing within a closed cage, followed by behavioral and energy metabolic analyses.

380 Our data indicated that optogenetic-elicited burst firing was sufficient to promote the

381 chronic stress-induced phenotypes described above. Taken together, these data show

382 that VMH burst firing is an important hub for integrating and interacting anxiety-like

383 emotional state and energy expenditure regulation.

\section{Mechanism of burst firing in dmVMH}

386 The mechanism underlying neuronal burst firing is complicated and could explain specific functions in different brain regions. Previous studies have reported that various modulatory substances can promote depolarization of membranes and evoke

389 burst firing of neurons. Oxytocin binds to its receptor in CA2 region and can activate

390 the G protein-coupled pathway, and thus induce activation of several spike channels

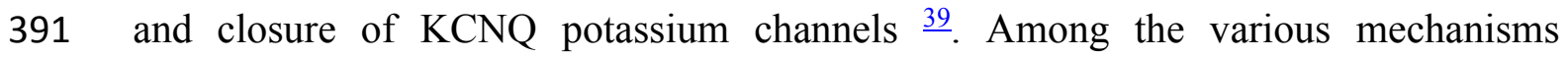

392 underlying burst initiation, activation of a low-threshold ion channel can induce burst

393 firing in diverse brain regions $\underline{26}, \underline{31}$. Typically, low-threshold T-VGCC is widely

394 expressed in the central nervous system and can be activated by stimuli near the RMP

395 to elicit burst firing. Molecular cloning has revealed three isoforms of T-type channel

396 genes: i.e., Cav3.1, Cav3.2, and Cav3.3, which make distinct contributions to cellular

397 electrical properties $\underline{31,32,42}$. Selective expression of Cav3.1 is sufficient to generate a 398 strong rebound burst in deep cerebellar nuclear neurons, whereas expression of 
399 Cav3.2 or Cav3.3 alone does not generate a rebound discharge under normal

400 conditions $\stackrel{31}{ }$. Several studies have demonstrated that substitution of Cav3.1 or Cav3.2

401 for the native channel in model thalamic relay neurons causes elimination of

402 high-frequency bursts, further confirming the role of Cav3.1 in burst firing $\underline{32,42}$.

403

404 Here, we identified the existence of Cav3.1, Cav3.2, and Cav3.3 in the dmVMH

region. Cav3.1 and Cav3.2 displayed high expression in the dmVMH, whereas Cav3.3

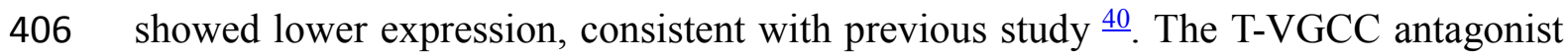

407 mibefradil blocked burst firing of dmVMH neurons, thus suggesting the existence of

408 T-VGCC-mediated burst firing in the dmVMH. Furthermore, combined analysis of

409 electrophysiological classification and single-cell qRT-PCR, we found that Cav3.1

410 expression increased under chronic stress, which contributed to the enhancement of

411 burst firing in the dmVMH. Furthermore, Cav3.1 demonstrated greater contribution to

412 stress-induced enhancement of bursting in the dmVMH than the other isoforms.

414 We further investigated the necessity of Cav3.1 in chronic stress-induced anxiety-like

415 behavior and energy metabolic disorders. We found that microinjection of mibefradil

416 ameliorated anxiety-like behavior. Using RNAi methods, the expression of Cav3.1 in

417 the dmVMH was specifically knocked down, resulting in the significant inhibition of

418 neuronal bursting activity. Behavioral and energy expenditure experiments further

419 demonstrated that the chronic stress responses described above were partially rescued. 
420 Thus, our findings consistently demonstrated that Cav3.1 may be a potential drug

421 target for the treatment of anxiety and related energy metabolic disorders.

422

423 The current study has several limitations. Firstly, we focused on exploring neuronal

424 activity changes in the dmVMH after chronic stress. As such, further research should

425 focus on identifying the mechanism regulating Cav3.1 expression in the dmVMH,

426 especially the receptors regulating Cav3.1 expression. Several factors might contribute

427 to the increased percentage of burst firing neurons after stress, including stress-induced

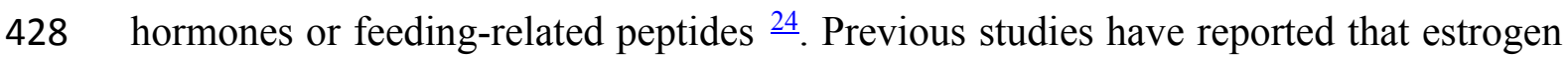

429 can regulate the expression and function of T-VGCC in vlVMH neurons, but not

$430 \mathrm{dmVMH}$ neurons, through estrogen receptors $\stackrel{40}{ }$. However, the mechanism underlying

431 Cav3.1 expression influenced by hormones or neuropeptides after chronic stress needs

432 further study.

433

434 Secondly, GABAergic neural circuits and astrocytes have been found in the VMH and

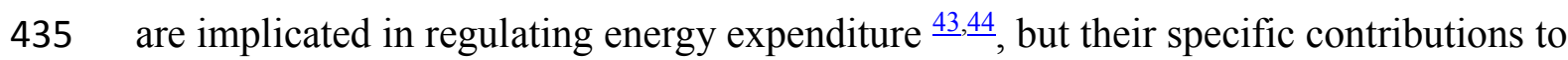

436 bursting activity need to be further investigated. We demonstrated that glutamate

437 receptors (especially NMDA receptors) affect the generation of burst firing; however,

438 whether synaptic glutamate uptake mediated by astrocytes affects burst firing requires

439 further exploration. Moreover, as the function of the T-VGCC is highly membrane

440 potential dependent, how upstream inputs integrate with the surrounding 
441 microenvironment to affect membrane potential, and hence regulate burst firing,

442 needs to be further explored.

443

444 In summary, our study first identified bursting firing neurons in the dmVMH as a hub

445 regulating emotional state and energy metabolic disorders. We also identified Cav 3.1

446 as the crucial regulator of bursting firing of dmVMH neurons. The results of this

447 molecular and electrophysiological study should provide a more complete

448 understanding of the chronic stress-induced emotional malfunction and peripheral

449 metabolism disorders, and provides potential therapeutic targets for treating such

450 malfunctions.

451 Competing interests

452 The authors declare no competing interests.

\section{Acknowledgements}

455 This project was partly supported by the National Natural Science Foundation of 456 China (81471164, 31800881), Key Research Program of Frontier Sciences of Chinese 457 Academy of Sciences (QYZDB-SSW-SMC056), and Shenzhen Governmental Basic 458 Research Grant (JCYJ20170413164535041, JCYJ20180507182301299). We also

459 thank Z.B Xu and B.F Liu for their help in transgenic mice husbandry and 460 phenotyping. We are grateful to N.N Li and X.L Liu for the help in virus packaging.

\section{Author contributions}

462 F.Y. and H.-L.H. conceived the idea and designed the experiments. J.S. D.-S.G and 
464 behavioral experiments, S.-P.C. and X.-Y.Z. helped with patch clamp recording and

465 qRT-PCR, L.Z. helped with immunostaining and surgery, N.L. helped with the

466 RNAi construction, Q.X. helped with in vivo electrophysiological recording, F.Y. and

467 J.S. interpreted the results and wrote the manuscript with critical inputs from H.-L.H.

468 and L.-P.W..

\section{$470 \quad$ Methods}

$471 \quad$ Animals

472 All procedures were carried out in accordance with protocols approved by the Ethics

473 Committee of the Shenzhen Institutes of Advanced Technology, Chinese Academy of

474 Sciences. Male C57BL/6 mice (4-8 weeks old) were purchased from the Guangdong

475 Medical Laboratory Animal Center (Guangzhou, China). The SF-1-Cre mice (stock no:

476 012462) were obtained from Jackson Laboratories (Bar Harbor, ME, USA). Mice were

477 housed at $22-25^{\circ} \mathrm{C}$ on a circadian cycle of 12-hour light and 12-hour dark with

478 ad-libitum access to food and water.

\section{Chronic stress procedures}

480 All animals used in this study were male, and randomly assigned to either control or

481 stress groups for chronic stress study. The stress group was daily subjected to one 482 stressor which randomly chosen from following: (i) 10 mice squeezing in a relatively 
487 Control animals were subjected to no stressors.

\section{Behavioral tests}

493 Videos recorded during the EPM test were analyzed with ANY-maze software

494 (Stoelting Co., Wood Dale, USA) to acquire data on time spent in open and closed arms,

495 locomotor activity (total distance travelled in maze), and entries into the open arm.

496 Anxiety-related behavior is associated with less exploration in the open arm relative to

497 overall exploration of all arms.

498 Open field test (OFT). Open field exposure (10-min) was used to assess locomotor 499 activity and anxiety-related behaviors after chronic stress. Mice ( $n=18-20$ /group)

500 were placed in the center of an open field and behavior was recorded for the entire 10

501 min. Videos were analyzed to acquire data on time spent in the center and corner areas,

502 total locomotor activity, and number of entries into the center area. Anxiety-related

503 behavior is associated with less exploration of the center area. 


\section{CLAMS and energy expenditure}

505 To characterize metabolic changes caused by chronic stress or Cav 3.1 knockdown,

506 RER was measured by indirect calorimetry using a four-chamber open-circuit

507 calorimeter (Oxymax Series; Columbus Instruments, Columbus, OH, USA). Mice were

508 food deprived overnight, with body weight and chow in each cage weighed prior to the

509 experiment. During the experiment, mice were housed individually in specially built

510 Plexiglas cages $(40 \times 25 \times 20 \mathrm{~cm})$. Temperature was maintained at $22{ }^{\circ} \mathrm{C}$ with an

511 airflow of 0.5 per min. Food and water were available ad libitum. Mice were

512 subsequently monitored in the system for $24 \mathrm{~h}$ (whole light-dark cycle). Oxygen

513 consumption (VO2) and carbon dioxide production (VCO2) were measured every 10

514 min. The RER was calculated as a quotient of $\mathrm{VCO} 2 / \mathrm{VO} 2$, with 1 representing $100 \%$

515 carbohydrate oxidation and 0.7 representing 100\% fat oxidation. Energy expenditure

516 (kcal heat produced) was calculated as calorific value $(\mathrm{CV}) \times \mathrm{VO} 2$, where $\mathrm{CV}$ is 3.815

$517+1.232 \times$ RER 3 . Metabolic data collected from the 24-h monitoring period were

518 averaged for energy expenditure and RER. After the experiment, chow in each cage

519 was weighed to calculate food intake.

\section{Slice preparation}

521 Mice were deeply anesthetized with isoflurane and decapitated rapidly. Brains were

522 then removed and transferred to chilled cutting solution within $3 \mathrm{~min}$. Cutting solution

523 contained (in $\mathrm{mM}$ ): choline chloride $110 ; \mathrm{KCl} 2.5 ; \mathrm{Na}$-pyruvate $0.6 ; \mathrm{MgCl}_{2} 7.0 ; \mathrm{CaCl}_{2}$ 
$524 \quad 0.5 ; \mathrm{NaH}_{2} \mathrm{PO}_{4} 1.3 ; \mathrm{NaHCO}_{3} 25 ;$ glucose $20(\mathrm{pH}$ 7.4). The chilled cutting solution was

525 bubbled with carbogen $\left(95 \% \mathrm{O}_{2}\right.$ and $\left.5 \% \mathrm{CO}_{2}\right)$ for at least $30 \mathrm{~min}$ before use. Coronal

526 slices (250-300 mm thick) were prepared on a vibratome (Series 1000, Warner

527 Instruments, Berlin, Germany), and incubated in artificial cerebrospinal fluid (ACSF)

528 containing (in $\mathrm{mM}$ ): $\mathrm{NaCl} 125 ; \mathrm{KCl} 2.5 ; \mathrm{Na}$-pyruvate $0.6 ; \mathrm{MgCl}_{2}$ 1.3; $\mathrm{CaCl}_{2} 2.0$;

$529 \mathrm{NaH}_{2} \mathrm{PO}_{4} 1.3 ; \mathrm{NaHCO}_{3} 25$; glucose $10(\mathrm{pH} 7.4)$ bubbled with carbogen at $34{ }^{\circ} \mathrm{C}$ for 30

530 min. After incubation, all slices were equilibrated in ACSF at room temperature

$531 \quad\left(24-26^{\circ} \mathrm{C}\right)$ for at least $40 \mathrm{~min}$. Single slices were placed on the recording chamber

532 perfused with ACSF bubbled with carbogen at room temperature. Unless stated

533 otherwise, drugs were applied with perfused ACSF.

\section{Electrophysiology}

535 Whole-cell patch clamp recording was performed on VMH neurons. The dmVMH was

536 identified based on landmarks (third ventricle). Recordings were obtained with

537 multi-clamp 700B amplifiers (Molecular Devices, San Jose, USA) under visual

538 guidance using a Nikon FN1 microscope (Tokyo, Japan). Electrophysiological data

539 were acquired and analyzed using pClamp 10 software (Molecular Devices, San Jose,

540 USA ). Whole-cell recordings were performed with borosilicate glass electrodes $(0.69$

$541 \mathrm{~mm} \mathrm{OD}, 5-7 \mathrm{M} \Omega$ ) with internal solution containing (in $\mathrm{mM}$ ): K-gluconate 135.0; $\mathrm{KCl}$

542 4.0; $\mathrm{NaCl}$ 2.0; HEPES 10; EGTA 4.0; Mg-ATP 4.0; Na-GTP 5.0. Osmolality was

543 adjusted to $290-310 \mathrm{mOsm} \mathrm{kg}^{-1}$ with sucrose and $\mathrm{pH}$ was adjusted to 7.4 with $\mathrm{KOH}$. 
545 After forming a high-resistance seal (G $\Omega)$, the cell was held in current-clamp mode for

$546 \quad 7-10$ min until access resistance stabilized. Resting membrane potential (RMP) was

547 assessed at the beginning of the recording period after stabilization of access resistance,

548 and periodically monitored throughout the recording by momentarily relieving the

549 direct current injection. To elucidate differences among neurons, 800-ms current

550 injections (-100 to $100 \mathrm{pA}$ in $10 \mathrm{pA}$ increments; $5 \mathrm{~s}$ interstimulus interval) were applied,

551 and the number of action potentials evoked by each current injection, input resistance,

552 and half-width of action potentials were determined.

\section{Drugs and reagents}

554 All chemicals included in ACSF prescription were purchased from Sigma-Aldrich

555 (Merck KGaA, Darmstadt, Germany). Mibefradil $(40 \mu \mathrm{M})$ were purchased from Tocris

556 (Bio-Techne, Minneapolis, USA) and used for electrophysiology and microinjection.

557 NBQX (1,2,3,4-tetrahydro-6-nitro-2,3-dioxo- benzo[f]quinoxaline-7-sulfonamide, 30

$558 \mu \mathrm{M})$ and AP5 (2-amino-5-phosphono-pentanoic acid, $30 \mu \mathrm{M})$ were acquired from Med

559 Chem Express (MCE, Shanghai, China).

560 Cell harvesting and single-cell Quantitative real-time polymerase chain reaction

561 (qRT-PCR)

562 Single Cell-to-CT ${ }^{\mathrm{TM}}$ Kits (Thermo Fisher, USA) were used to perform single-cell

563 qRT-PCR. After recording, single cells were collected and lysed to acquire total RNA.

564 A Mastercycler 5333 PCR thermal cycler (Eppendorf, Germany) was used to perform 
567 housekeeping gene Rn18s. All protocols were performed according to the

568 manufacturer's instructions. qRT-PCR was performed with a Light cycler 480 (Roche,

569 Switzerland).

\section{Tissue collection and qRT-PCR}

571 To acquire dmVMH tissue, mice were first anesthetized with isoflurane. After bathing

572 in cold 1\% diethyl pyrocarbonate (DEPC) phosphate-buffered saline (PBS) solution,

573 brains were acute cut on a vibratome. VMH tissues were dissected microscopically

574 from those sections, and quickly transferred to TRIzol reagent. The manufacturer's

575 standard protocols for RNA extraction (TransGene, China) and synthesis (TOYOBO,

576 Japan) were followed. Primers used for qRT-PCR included: Cav 3.1 (5'-TGG

577 CCTTCTTCGTCCTGAAC-3' and 5'-TTCTCCAGCCTCTTTAGTCGC-3'). Cav 3.2

578 (5'-CGGCCCTACT ACGCAGACTA-3' and 5'-TTAAGGGCCTCGTCCAGAGA-3'),

579 Cav 3.3 (5'-CTGCTATTCTCCAGCCCAGG-3' and 5'-AGCTGCACCTCTTG

580 CTTGT-3'). Expression of these gene was normalized to the expression of

581 housekeeping gene $\beta$-Actin $(A c t-b)$.

\section{In vivo electrophysiology}

583 Mice were implanted with two nickel-chromium wires $(25-\mu \mathrm{m}$ diameter; AM Systems,

584 Sequim, USA) targeting the dmVMH, connector was bind to wires and fixed on skull

585 with dental cement. After total recovery from surgery, mice were returned to the 


\section{Stereotaxic surgery and viral injection}

593 For all stereotaxic surgery, 12-16-week-old mice were anesthetized by pentobarbital

594 sodium $(0.3 \%$ in saline, $1 \mathrm{ml} / 100 \mathrm{~g}$, intraperitoneally) and placed in a stereotaxic

595 apparatus for surgery. Stereotaxic surgical procedures were performed using standard

596 protocols. To target the dmVMH, bilateral brain injection coordinates relative to

597 bregma were chosen (AP, $-1.58 \mathrm{~mm}$; ML, $\pm 0.3 \mathrm{~mm}$; DV, $-5.5 \mathrm{~mm}$ ). Unless stated

598 otherwise, $0.4 \mu \mathrm{L}$ of viral vector was injected into the $\mathrm{VMH}$ at a rate of $0.1 \mu \mathrm{L} / \mathrm{min}$ 599 using a 10- $\mu \mathrm{L}$ Hamilton syringe and a syringe infusion pump (World Precision 600 Instruments, USA).

601

603 was injected into the VMH with lenti-viral vector. Mice were housed for four weeks

604 following injection for viral expression. Anxiety-like behavior and metabolic tests were

605 performed at the end of the paradigm. To assess the knockdown effectiveness of Cav

6063.1 shRNA, mice were perfused with 4\% paraformaldehyde (PFA) and brain tissues 
607 were removed for immunostaining analysis after the final session.

608 For microinjection, a stainless-steel guide-cannula $(0.6 \mathrm{~mm}$ outer diameter and $0.4 \mathrm{~mm}$

609 inner diameter, RWD, Shenzhen, China) was implanted into the diencephalon to target

610 the VMH (AP, $-1.58 \mathrm{~mm}$; ML, $\pm 0.3 \mathrm{~mm}$; DV, $-5.0 \mathrm{~mm}$ ). The guide-cannula was fixed to

611 the skull using dental cement and three stainless steel screws. Each guide-cannula was

612 sealed with a stainless-steel wire for protection from obstruction. Before behavioral

613 testing, the stainless-steel wire was replaced with an injection cannula $(0.5 \mathrm{~mm}$ deeper

614 than guide-cannula to break astrocyte aggregation), through which the drug dissolved

615 in saline $(400 \mathrm{nl})$ was delivered at $100 \mathrm{nl} / \mathrm{min}$ into the VMH. After microinjection, mice

616 were rested for $30 \mathrm{~min}$ before tests.

617 Wireless optogenetic manipulation

618 To achieve wireless optogenetic manipulation of burst firing neurons, AAV9-DIO

619 -NpHR-mCherry was injected unilaterally into the VMH in the left hemisphere. Mice

620 were housed for four weeks following injection for viral expression before initiation of

621 experiments. A copper coil with a light emitting diode (LED) on the right side of the tip

622 (Inper, Hangzhou, China) was implanted into the brain of NpHR-expressing mice, with

623 the LED located $0.2 \mu \mathrm{m}$ left of the dmVMH. The coil and indicator were fixed on the

624 surface of the skull bone with Vetbond Tissue Adhesive (3M, USA). Charging the coil

625 was achieved through antennas surrounding the cage. To initiate burst firing of

$626 \mathrm{dmVMH}$ neurons, $550-\mathrm{nm}$ yellow light stimulation was performed at $0.1 \mathrm{~Hz}(2 \mathrm{~s} /$ pulse $)$

627 in the NpHR-mCherry group during the metabolic (one illumination trial/12 h, each 
trial lasted for $2 \mathrm{~h}$ ) and behavioral tests (OFT, $10 \mathrm{~min}$; EPM, $5 \mathrm{~min}$ ).

629

630

631

Slice electrophysiological data were analyzed with pCLAMP (Molecular Device, San

\section{Immunohistochemistry}

Mice were first anesthetized by chloral hydrate $(10 \%$ in saline, $1 \mathrm{ml} / 100 \mathrm{~g}$, intraperitoneally), then perfused with $0.01 \mathrm{M}$ PBS and $50 \mathrm{~mL}$ of $4 \%$ PFA transcardially. Brains were dissected and post-fixed in 4\% PFA overnight and then transferred to $30 \%$ $\mathrm{w} / \mathrm{v}$ sucrose solution for cryoprotection until sinking. Sections from the entire anterior-posterior range of the VMH were stained using an antibody specific to Cav3.1 (1: 100, Alomone labs, Cat\# ACC-021, RRID: AB_2039779), Cav3.2 (1: 100, Alomone labs, Cat\#ACC-025, RRID: AB_2039781), or Cav 3.3(1: 100, Alomone labs, Cat\# ACC-009, RRID: AB_2039783). Briefly, sections were washed, permeabilized in $0.1 \%$ Triton $\mathrm{X}-100 / \mathrm{PBS}$ for $15 \mathrm{~min} /$ three times, washed again, and blocked in $10 \%$ normal goat serum (NGS) (w/v)/0.1\% Triton X-100/PBS for 30 min. Primary antibody was added, and sections were incubated overnight at $4{ }^{\circ} \mathrm{C}$. The following day, sections were washed with $0.01 \mathrm{mM}$ PBS, incubated with incubated with secondary antibody (Alexa Fluor 594 Goat Anti-Rabbit, Cat\#115-585-003, RRID: AB_2338059 or Alexa Fluor 488 Goat Anti-Rabbit, Cat\#111-547-003 RRID: AB_2338058) in 1\% NGS/0.1\% Triton X-100/PBS for $1 \mathrm{~h}$ at room temperature, then washed, mounted, and cover slipped with mounting medium containing DAPI.

\section{Statistical analysis}

Jose, CA, USA). In vivo electrophysiology data were analyzed with NeuroExplorer 5.0 
649 (Plexon, Dallas, USA). All data were imported into Prism 7 (GraphPad Software, La

650 Jolla, CA, USA) and normality was assessed using D’Agostino-Pearson tests to verify

651 the appropriateness of the following statistical analyses. Unless stated otherwise, the

652 data are presented as means \pm SEM. Statistical significance was determined using

653 two-tailed unpaired Student's $t$-tests when comparing two groups, paired Student's

$654 t$-test when comparing the effects of different treatments in the same group. When

655 multiple measures were compared between groups (e.g., current-frequency curves),

656 repeated measures two-way analysis of variance (ANOVA) with Bonferroni's test was

657 used. Differences were considered significant at $P<0.05$.

658

659 To classify dmVMH neurons, unsupervised cluster analysis was performed with SPSS

660 v19 (Chicago, IL, USA) using squared Euclidean distances. The parameters for cluster

661 analysis were chosen based on their lack of linear correlation with each other. The

662 following electrophysiological parameters were included for analysis: onset time,

663 evoked firing rate, input resistance, and potential sag by H-current

664 (Hyperpolarization-activated current). Resting potential were excluded from the

665 parameters for cluster analysis because of their linear correlation with onset times. All

666 electrophysiological parameters were converted into standardized z-scores before

667 clustering. 


\section{References}

6711 Koolhaas, J. M. et al. Coping styles in animals: current status in behavior and

672 stress-physiology. Neuroscience \& Biobehavioral Reviews 23, 925-935,

6742 Viskaitis, P. et al. Modulation of SF1 Neuron Activity Coordinately Regulates Both Feeding Behavior and Associated Emotional States. Cell Reports 21, 3559-3572, doi:https://doi.org/10.1016/i.celrep.2017.11.089 (2017).

678

679 Hardaway, J. A. et al. Central Amygdala Prepronociceptin-Expressing Neurons Mediate Palatable Food Consumption and Reward. Neuron 102, 1088, doi:https://doi.org/10.1016/i.neuron.2019.04.036 (2019).

683 Neuroscience 5, 545-552, doi:10.1038/nrn1429 (2004). Reviews Neuroscience 10, 434-445, doi:10.1038/nrn2639 (2009). 5482-5490, doi:10.1210/en.2008-0642 (2008).

7 Isingrini, E. et al. Resilience to chronic stress is mediated by noradrenergic regulation of dopamine neurons. Nature Neuroscience 19, 560-563, doi:10.1038/nn.4245 (2016).

Kuperman, Y. et al. Perifornical Urocortin-3 mediates the link between stress-induced anxiety and energy homeostasis. Proceedings of the National Academy of Sciences of the United States of America 107, 8393-8398, doi:10.1073/pnas.1003969107 (2010).

703

Cheung, C. C. et al. Sex-dependent changes in metabolism and behavior, as well as reduced anxiety after eliminating ventromedial hypothalamus excitatory output. Molecular metabolism 4, 857-866, doi:10.1016/j.molmet.2015.09.001 (2015). stress and depression/anxiety-related behaviors. Molecular psychiatry 22, 1056-1068, doi:10.1038/mp.2016.225 (2017). CNS in the regulation of glucose homeostasis. Nature reviews. Neuroscience 14, 24-37, doi:10.1038/nrn3409 (2013). Silva, B. A. et al. Independent hypothalamic circuits for social and predator fear. Nature Neuroscience 16, 1731-1733, doi:10.1038/nn.3573 (2013).

13 Wang, L., Chen, I. Z. \& Lin, D. Collateral pathways from the ventromedial hypothalamus mediate defensive behaviors. Neuron 85, 1344-1358, doi:10.1016/j.neuron.2014.12.025 (2015).

14 Coutinho, E. A. et al. Activation of SF1 Neurons in the Ventromedial 
Hypothalamus by DREADD Technology Increases Insulin Sensitivity in Peripheral Tissues. Diabetes 66, 2372-2386, doi:10.2337/db16-1344 (2017).

15 Yadav, V. K. et al. A serotonin-dependent mechanism explains the leptin regulation of bone mass, appetite, and energy expenditure. Cell 138, 976-989, doi:10.1016/j.cell.2009.06.051 (2009).

16 McClellan, K. M., Parker, K. L. \& Tobet, S. Development of the ventromedial nucleus of the hypothalamus. Frontiers in Neuroendocrinology 27, 193-209, doi:https://doi.org/10.1016/i.yfrne.2006.02.002 (2006).

17 Anderson, D. J. Circuit modules linking internal states and social behaviour in flies and mice. Nature Reviews Neuroscience 17, 692, doi:10.1038/nrn.2016.125 (2016).

18 Hashikawa, K. et al. Esr1+ cells in the ventromedial hypothalamus control female aggression. Nat Neurosci 20, 1580-1590, doi:10.1038/nn.4644 (2017).

19 Kim, D. W. et al. Multimodal Analysis of Cell Types in a Hypothalamic Node Controlling Social Behavior. Cell 179, 713-728.e717, doi:10.1016/j.cell.2019.09.020 (2019).

20 Wang, C., Bomberg, E., Billington, C. J., Levine, A. S. \& Kotz, C. M. Brain-derived neurotrophic factor (BDNF) in the hypothalamic ventromedial nucleus increases energy expenditure. Brain research 1336, 66-77, doi:https://doi.org/10.1016/j.brainres.2010.04.013 (2010).

21 Klöckener, T. et al. High-fat feeding promotes obesity via insulin receptor/PI3K-dependent inhibition of SF-1 VMH neurons. Nature Neuroscience 14, 911-918, doi:10.1038/nn.2847 (2011).

22 Kunwar, P. S. et al. Ventromedial hypothalamic neurons control a defensive emotion state. elife 4, doi:10.7554/eLife.06633 (2015).

23 ligaya, K., Okazaki, S., Minoura, Y. \& Onimaru, H. Interaction between novel oscillation within the ventromedial hypothalamus and the sympathetic nervous system. Neuroscience 343, 213-221, doi:10.1016/j.neuroscience.2016.11.048 (2017).

24 ligaya, K., Minoura, Y., Onimaru, H., Kotani, S. \& Izumizaki, M. Effects of Feeding-Related Peptides on Neuronal Oscillation in the Ventromedial Hypothalamus. J Clin Med 8, 292, doi:10.3390/jcm8030292 (2019).

25 Wang, G. et al. CaV3.2 calcium channels control NMDA receptor-mediated transmission: a new mechanism for absence epilepsy. Genes \& development 29, 1535-1551, doi:10.1101/gad.260869.115 (2015).

26 Cain, S. M. et al. CaV3.2 drives sustained burst-firing, which is critical for absence seizure propagation in reticular thalamic neurons. Epilepsia 59, 778-791, doi:10.1111/epi.14018 (2018).

Yuan, Y. et al. Reward Inhibits Paraventricular CRH Neurons to Relieve Stress. Current Biology 29, 1243-1251.e1244, doi:10.1016/j.cub.2019.02.048 (2019). Yang, Y. et al. Ketamine blocks bursting in the lateral habenula to rapidly relieve depression. Nature 554, 317-322, doi:10.1038/nature25509 (2018). 
760

761

762

763

764

765

766

767

768

769

770

771

772

773

774

775

776

777

778

779

780

781

782

783

784

785

786

787

788

$789 \quad 40$

790

791

792

793

794

Lee, S., Lee, C., Woo, C., Kang, S. J. \& Shin, K. S. Chronic social defeat stress-induced enhancement of T-type calcium channels increases burst-firing neurons in the ventral subiculum. Biochemical and biophysical research communications 508, 1182-1187, doi:10.1016/j.bbrc.2018.12.073 (2019).

30 Lisman, J. E. Bursts as a unit of neural information: making unreliable synapses reliable. Trends Neurosci 20, 38-43, doi:10.1016/s0166-2236(96)10070-9 (1997).

31 Molineux, M. L. et al. Specific T-type calcium channel isoforms are associated with distinct burst phenotypes in deep cerebellar nuclear neurons. Proceedings of the National Academy of Sciences of the United States of America 103, 5555-5560, doi:10.1073/pnas.0601261103 (2006).

32 McRory, J. E. et al. Molecular and functional characterization of a family of rat brain T-type calcium channels. The Journal of biological chemistry 276, 3999-4011, doi:10.1074/jbc.M008215200 (2001).

33 Nelson, M. T. et al. Molecular mechanisms of subtype-specific inhibition of neuronal T-type calcium channels by ascorbate. The Journal of neuroscience : the official journal of the Society for Neuroscience 27, 12577-12583, doi:10.1523/jneurosci.2206-07.2007 (2007).

34 Sherman, S. M. Tonic and burst firing: dual modes of thalamocortical relay. Trends in Neurosciences 24, 0-126 (2001).

35 Cui, Y. et al. Astroglial Kir4.1 in the lateral habenula drives neuronal bursts in depression. Nature 554, 323-327, doi:10.1038/nature25752 (2018).

36 Cain, S. M. et al. GABAB receptors suppress burst-firing in reticular thalamic neurons. Channels (Austin, Tex.) 11, 574-586, doi:10.1080/19336950.2017.1358836 (2017).

37 Matott, M. P., Kline, D. D. \& Hasser, E. M. Glial EAAT2 regulation of extracellular nTS glutamate critically controls neuronal activity and cardiorespiratory reflexes. The Journal of physiology 595, 6045-6063, doi:10.1113/jp274620 (2017).

38 Steffens, A. B., Scheurink, A. J. W., Luiten, P. G. M. \& Bohus, B. Hypothalamic food intake regulating areas are involved in the homeostasis of blood glucose and plasma FFA levels. Physiology \& Behavior 44, 581-589, doi:https://doi.org/10.1016/0031-9384(88)90322-8 (1988).

39 Tirko, N. N. et al. Oxytocin Transforms Firing Mode of CA2 Hippocampal Neurons. Neuron 100, 593-608.e593, doi:10.1016/j.neuron.2018.09.008 (2018).

40 Qiu, J. et al. Estrogen upregulates T-type calcium channels in the hypothalamus and pituitary. The Journal of neuroscience : the official journal of the Society for Neuroscience 26, 11072-11082, doi:10.1523/jneurosci.3229-06.2006 (2006).

41 Berglind, F. et al. Optogenetic inhibition of chemically induced hypersynchronized bursting in mice. Neurobiology of Disease 65, 133-141, 
doi:https://doi.org/10.1016/i.nbd.2014.01.015 (2014).

42 Cain, S. M. \& Snutch, T. P. Contributions of T-type calcium channel isoforms to neuronal firing. Channels 4, 475-482, doi:10.4161/chan.4.6.14106 (2010).

43 Bouyakdan, K. et al. The gliotransmitter ACBP controls feeding and energy homeostasis via the melanocortin system. The Journal of clinical investigation 129, 2417-2430, doi:10.1172/jci123454 (2019).

801

44 Chan, O. et al. Increased GABAergic Output in the Ventromedial

802 Hypothalamus Contributes to Impaired Hypoglycemic Counterregulation in

803 Diabetic Rats. Diabetes 60, 1582-1589, doi:10.2337/db10-1579 (2011).

804 
a

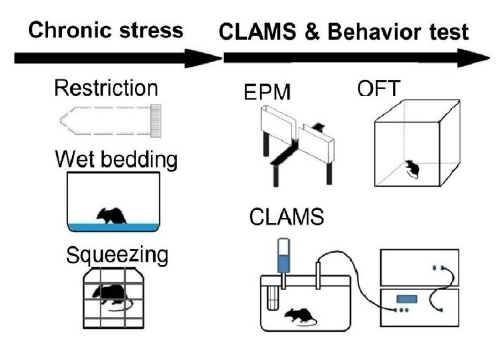

f

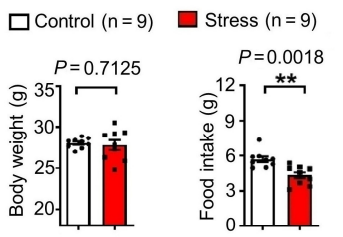

g

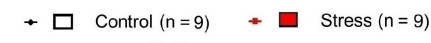

RER

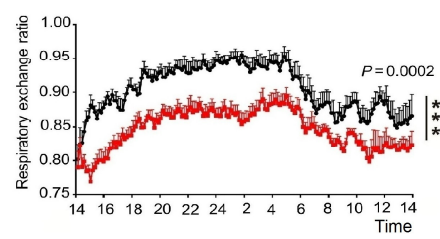

i

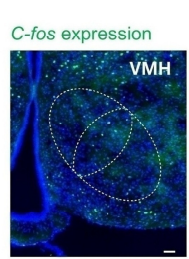

Control

k
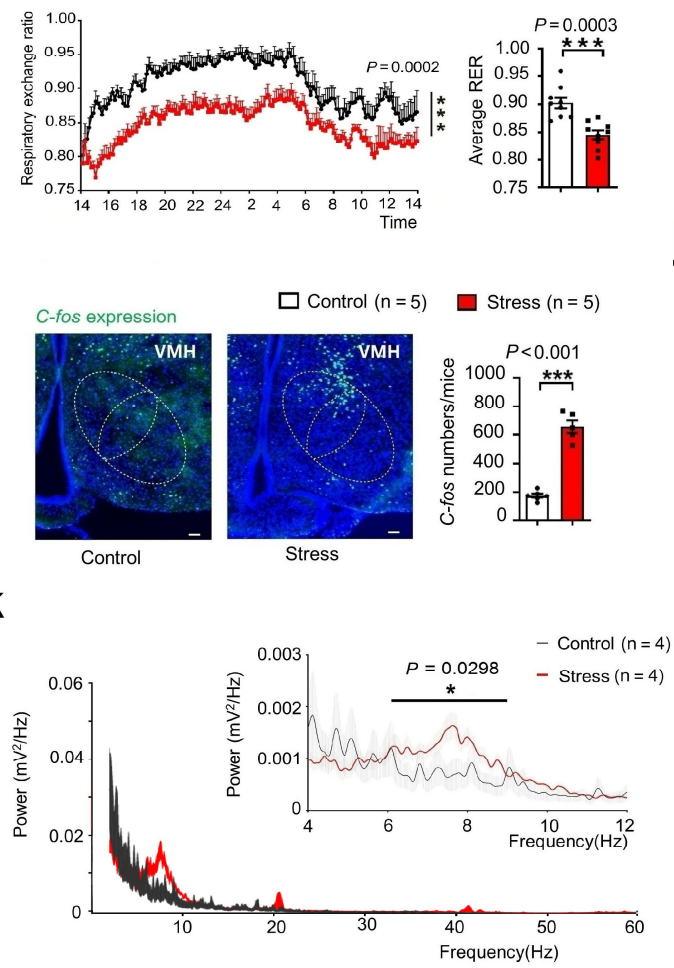

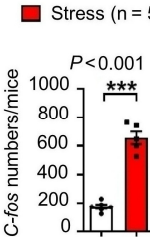

b

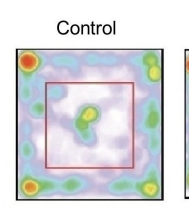

d

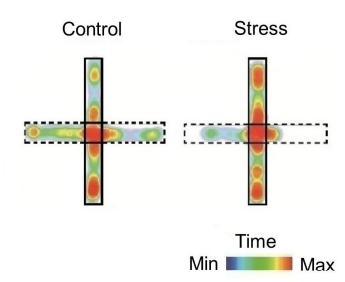

C

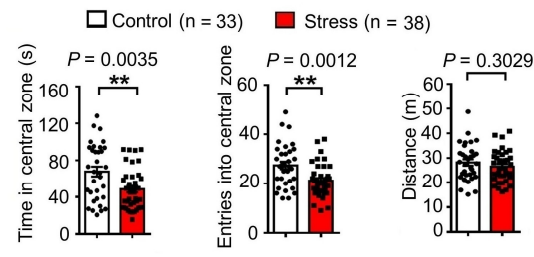

e

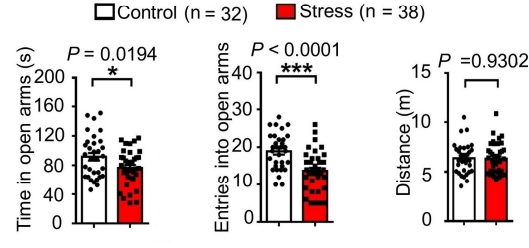

h

h $\quad \square$ Control $(n=9) \quad-\quad$ Stress $(n=9)$

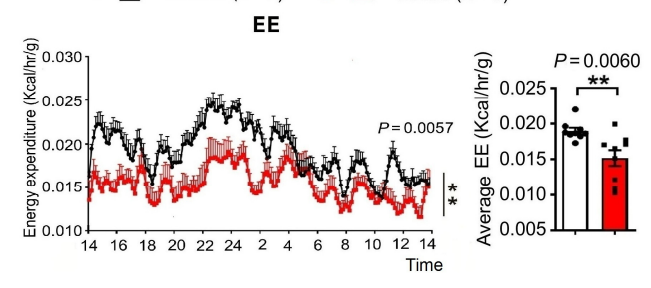

j

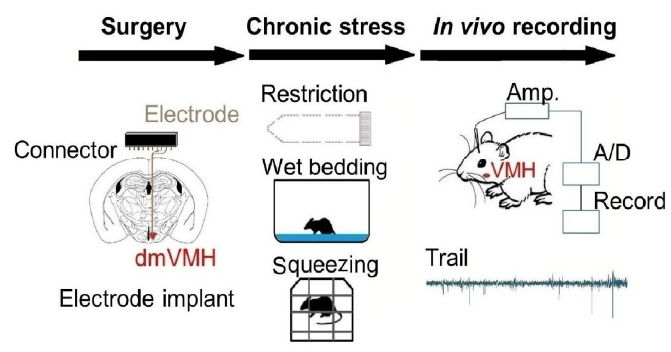

I

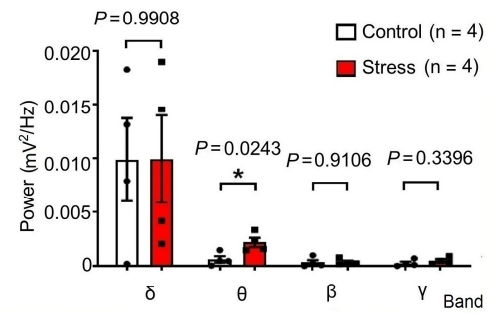

Fig. 1| Chronically stressed mice exhibited anxiety-like behavior, altered

(a) Illustration of unpredictable chronic stress protocol and phenotype assessment, with 
812 time in each site of open field (blue, less time; red, more time). (c) Behavioral analysis

813 of control $(n=33)$ and stress group mice $(n=38)$ in open field test showed significant

814 decrease in both time spent in central area (unpaired Student's $t$-test, $P=0.0035$ ) and

815 entries into central area (unpaired Student's $t$-test, $P=0.0012$ ), but no obvious change

816 in travelling distance (unpaired Student's $t$-test, $P=0.3029$ ). (d) Residence time in each

817 site of elevated plus-maze (blue, less time; red, more time) of control and stress groups.

818 (e) Control $(\mathrm{n}=32)$ and stress groups $(\mathrm{n}=38)$ in elevated plus-maze showed significant

819 decrease in time spent in open arm (unpaired Student's $t$-test, $P=0.0194$ ) and entries

820 into open arm (unpaired Student's $t$-test, $P<0.0001$ ), but no obvious change in

821 locomotion (unpaired Student's $t$-test, $P=0.9302$ ). (f) Body weight monitored after

822 chronic stress period showed no obvious change compared with naïve mice $(n=9$ in

823 each group, unpaired Student's $t$-test, $P=0.7125$ ), and 24-h food intake after overnight

824 fasting decreased in stress group ( $\mathrm{n}=9$ in each group, unpaired Student's $t$-test, $P=$

$8250.0018)$ compared with control group $(\mathrm{n}=9)$. $(\mathrm{g})$ Average respiration exchange ratio

826 (RER) decreased in stress group compared with control group (unpaired student's $t$-test,

$827 P=0.0003, \mathrm{n}=9$ mice in each group); RER curve shifted after chronic stress (two-way

828 ANOVA, $P=0.0002, F(1,16)=21.95)$. (h) Significant decreases in 24-h energy

829 expenditure (EE) curve and average EE were observed in stress group (two-way

830 ANOVA, $P=0.0057, F(1,16)=20.2$; unpaired Student's $t$-test, $P=0.0060)$. (i)

831 Increased $c$-fos expression in dmVMH under chronic stress (unpaired Student's $t$-test, $P$

$832<0.001, \mathrm{n}=5$ mice in each group). (j) Schematic of electrode implantation and in vivo 
833 electrophysiology recordings. (k and $\mathbf{l})$ Power spectral density of local field potential

834 (LFP) in dmVMH after chronic stress, with significant power improvement observed in

835 theta band (two-way ANOVA, $P=0.0298, F(1,14)=5.845$; ; unpaired Student's $t$-test,

$836 P=0.0243, \mathrm{n}=4)$. Data are means \pm SEM. $* P<0.05, * * P<0.01, * * * P<0.001$.

837 CLAMS, comprehensive laboratory animal monitoring system.

838 
a
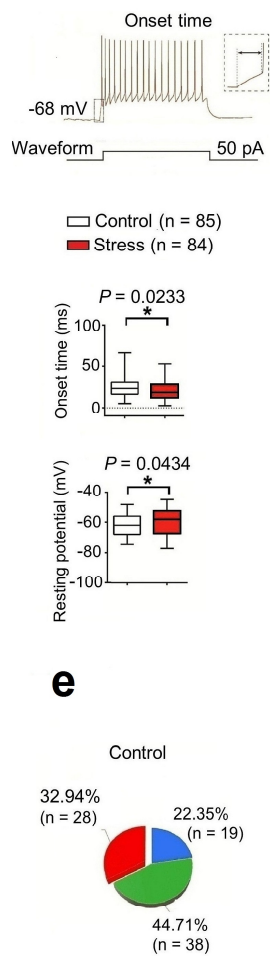

g
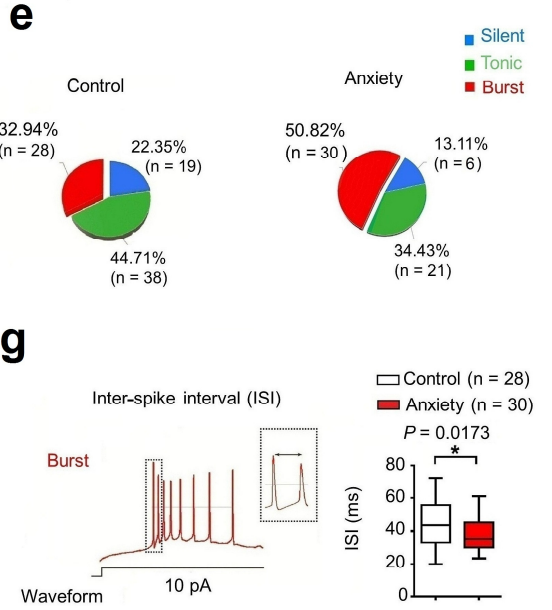

b

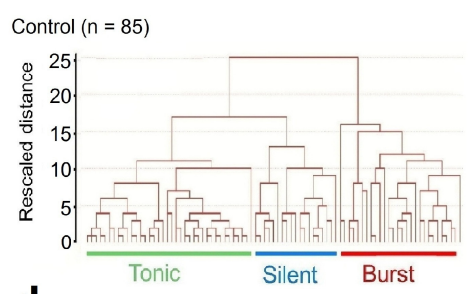

d

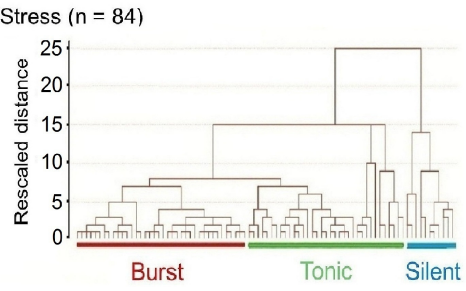

C
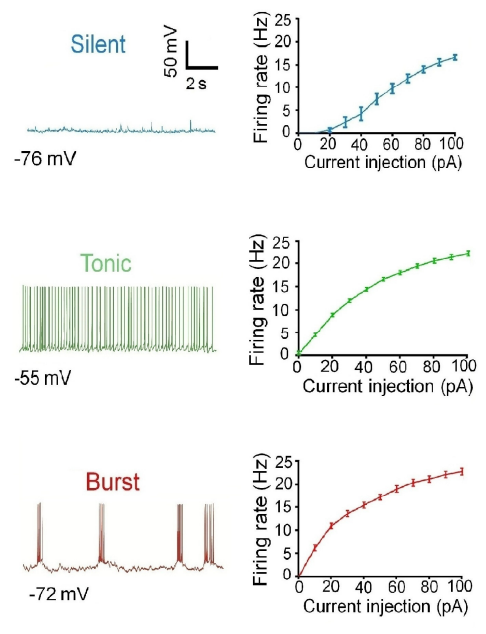

f
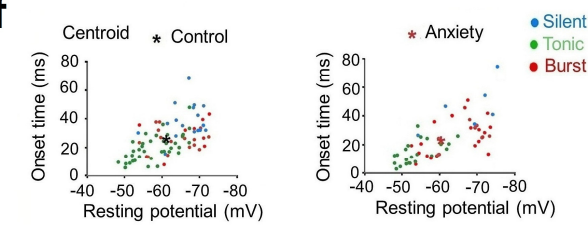

h

i

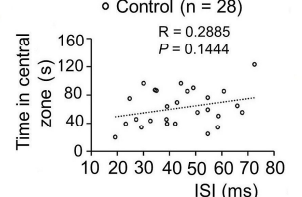

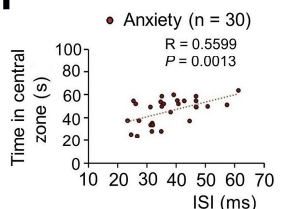
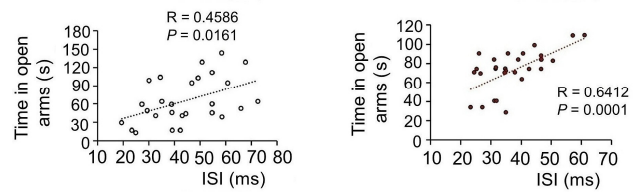

841 (a) Increased average onset time (unpaired Student's $t$-test, $P=0.0233$ ) and depolarized

842 average resting membrane potential (RMP) (unpaired Student's $t$-test, $P=0.0434$ ) in 84

$843 \mathrm{dmVMH}$ neurons from stressed mice compared with 85 neurons from wild-type mice.

844 The box plotted at the median extending from the 25-75th percentile, and the whisker represents Min to Max distribution. (b) Cluster analysis of $85 \mathrm{dmVMH}$ neurons from 35 normal mice. Dendrogram of cluster analysis shows that dmVMH neurons could be classified into three subtypes: i.e., silent, tonic-firing, and bursting. 
848 Electrophysiological properties of silent, tonic-firing, and bursting dmVMH neuronal

849 subtypes. left: whole-cell recording traces of three neuronal subtypes without current

850 injection; right: frequency-current curve of three subtypes at current injections of 0 to

$851100 \mathrm{pA}$ and $10 \mathrm{pA} / \mathrm{step}$. (d) Cluster analysis of $84 \mathrm{dmVMH}$ neurons from 39 stressed

852 mice. Dendrogram of cluster analysis shows these dmVMH neurons can be classified

853 into three subtypes: i.e., silent $(n=13)$, tonic-firing $(n=35)$, and bursting $(n=38)$. See

854 also Figure S1 and S2. (e) Pie chart of percentages of neuronal dmVMH subtypes in

855 control group and stressed mice with obvious anxiety-like behavior (anxiety group). (f)

856 Distribution of $85 \mathrm{dmVMH}$ neurons in control mice (left) and $57 \mathrm{dmVMH}$ neurons in

857 anxiety group (right) using onset time-RMP coordinate system, with coordinate of

858 centroid ( $\star$ ) indicating average onset time and RMP. Blue, silent; Green, tonic-firing;

859 Red, bursting. Centroid coordinate was determined by average onset time and RMP.

860 Shift in centroid after chronic stress represents shorter average onset time and more

861 depolarized average RMP, caused by changes in proportion of three subtypes. (g)

862 Inter-spike interval (ISI) of bursts in dmVMH neurons of control and stressed mice.

863 Left, Example of burst firing and ISI; right, ISI of burst firing dmVMH neurons $(\mathrm{n}=30)$

864 in anxiety group decreased significantly compared with that in control group $(\mathrm{n}=28$,

865 unpaired Student's $t$-test, $P=0.0099$ ). (h) ISI of dmVMH bursting neurons in control

866 group is slightly correlated with the residing time in open arms of EPM, but not with

867 the time spent in central area of open field $(n=28$ cells from 21 mice). (i) ISI of

$868 \mathrm{dmVMH}$ bursting neurons in stressed mice which displayed obvious anxiety behavior 
869 is significantly correlated with both the residing time in open arms and the time in

870 central area of open field $(\mathrm{n}=30$ cells from 20 mice). Data are means $\pm \mathrm{SEM} * P<$

$8710.05, * * P<0.01$. 
a

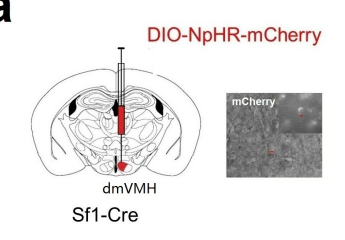

C
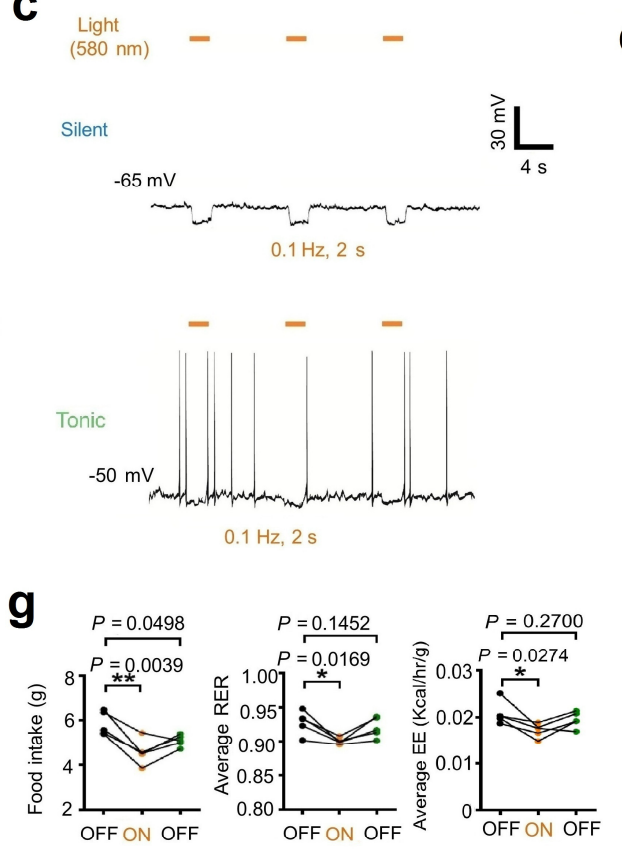

h

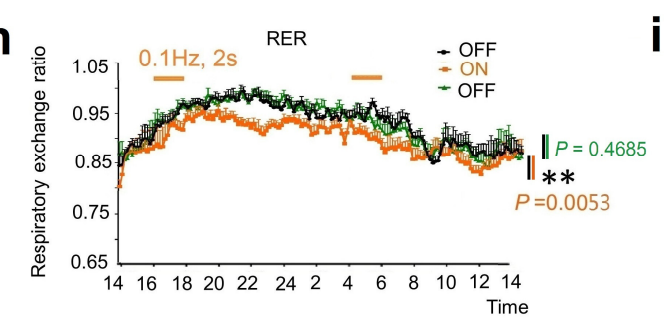

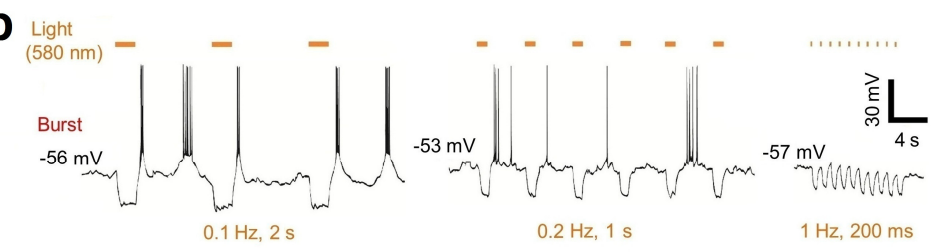

d
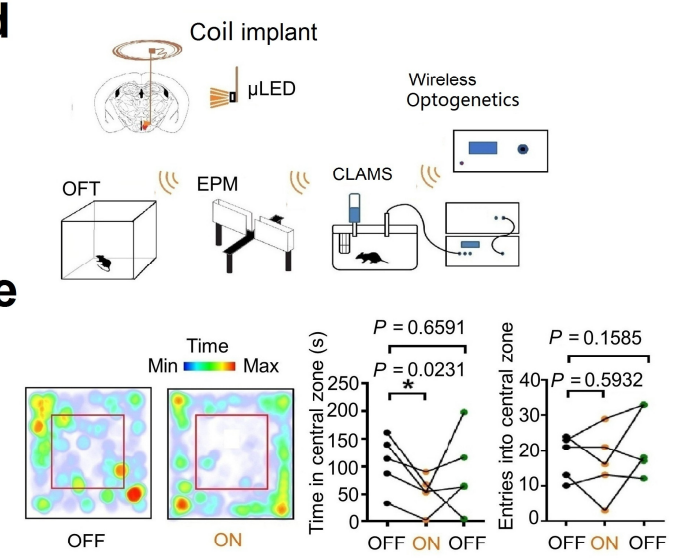

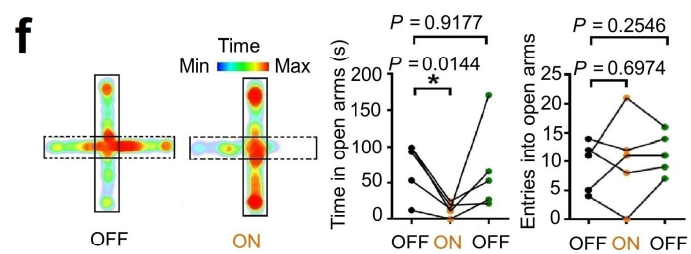

873 Fig. 3| Optogenetic activation of burst firing neurons in dmVMH induced anxiety-like behavior and energy expenditure changes.

875 (a) Schematic of dmVMH injection of NpHR AAV viral vector to induce burst firing in vivo. (b) Whole-cell recordings of yellow light-evoked burst firing in brain slices

877 (yellow light: $590 \mathrm{~nm}$, left: $0.1 \mathrm{~Hz}, 2 \mathrm{~s}$; middle: $0.2 \mathrm{~Hz}, 1 \mathrm{~s}$; right: $1 \mathrm{~Hz}, 200 \mathrm{~ms}$ ), $0.1 \mathrm{~Hz}$ 878 successfully induced activation of burst firing neurons. (c) $0.1 \mathrm{~Hz}$ and $2 \mathrm{~s}$ yellow light 879 illumination exerted no significant influence on silent or tonic-firing dmVMH neurons. (d) Illustration of wireless optogenetic manipulation of dmVMH neurons and 
881 behavioral analysis in free-moving mice. (e) Open field test before, during, and after

882 light illumination: residence time in central area decreased during 10-min yellow light

883 illumination ( $\mathrm{n}=5$, paired Student's $t$-test, $P=0.0237$ ), though no obvious changes

884 were observed in number of entries into central area (paired Student's $t$-test, $P=$

885 0.5932). (f) Elevated plus-maze test before, during, and after 5-min light illumination.

886 Residence time in open arms decreased during yellow light illumination $(n=5$, paired

887 Student's $t$-test, $P=0.0144$ ) and recovered after light-off; no obvious changes were

888 observed in number of entries into open arms (paired Student's $t$-test, $P=0.6974)$. (g)

889 Food intake and metabolism were monitored during optogenetic manipulation of

$890 \mathrm{dmVMH}$ neurons in free-moving mice. Food intake decreased during light stimulation

891 (paired Student's $t$-test, $P=0.0039, \mathrm{n}=5$ mice in each group), also average RER

892 (paired Student's $t$-test, $P=0.0169$ ) and EE (paired Student's $t$-test, $P=0.0274$ ). (h and

893 i) RER and EE curve shifted (two-way ANOVA, RER: $P=0.0053, F(1,8)=14.38$; EE:

$894 P=0.0227, F(1,8)=7.7918)$ when applying two yellow light stimulation trials $(0.1 \mathrm{~Hz}$,

$8952 \mathrm{~s}$; for $2 \mathrm{~h})$. Data are means $\pm \mathrm{SEM}$; ${ }^{*} P<0.05, * * P<0.01$. 
a
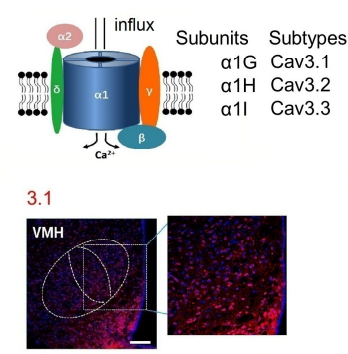

d

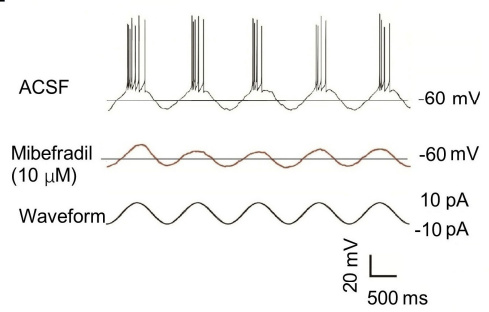

f

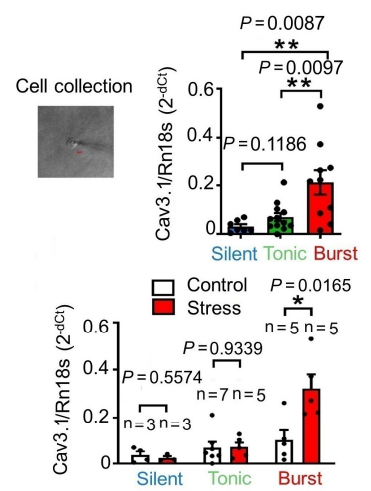

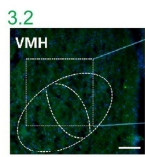

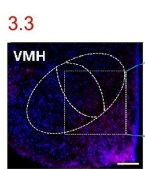

e
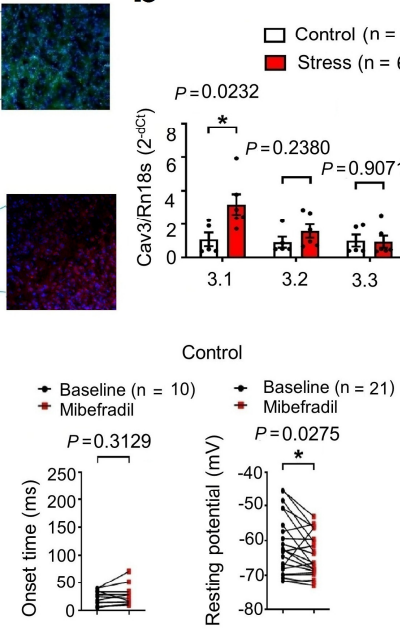

b
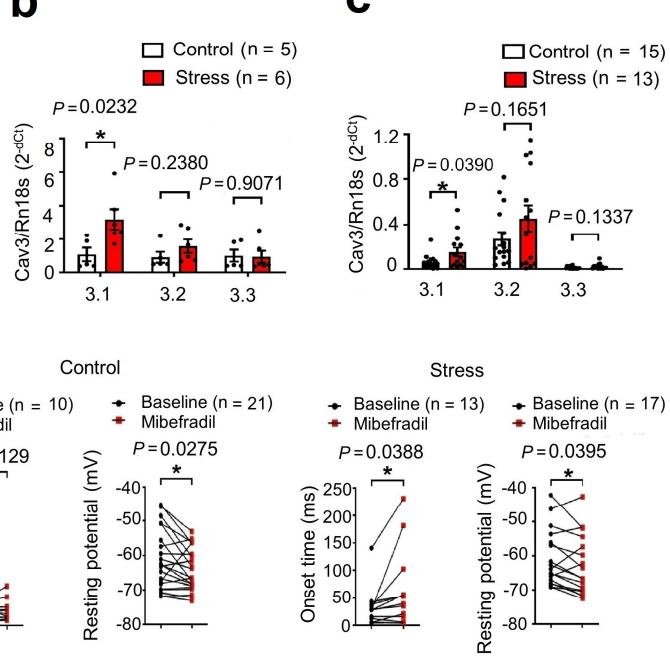

Control Burst

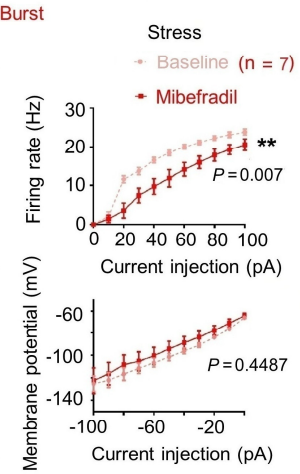

h
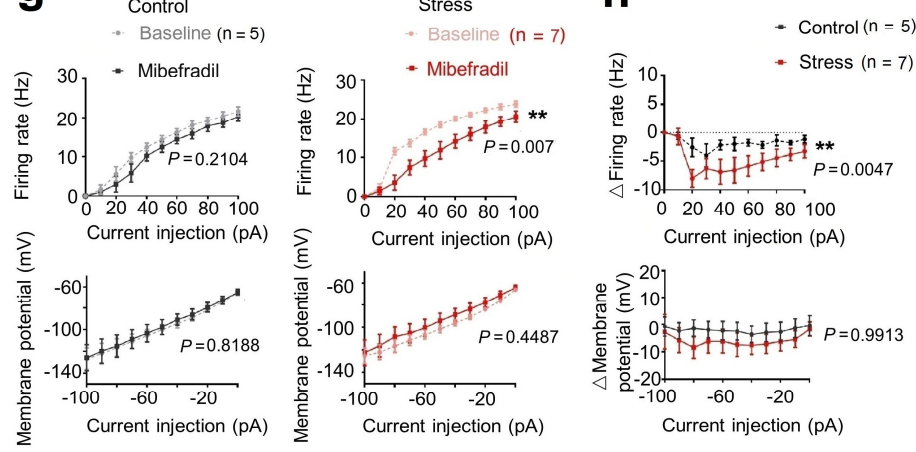

Fig. 4| T-VGCC mediated enhancement of burst firing in dmVMH neurons under

\section{chronic stress.}

(a) Schematic of structure of T-VGCC located on cell membrane (left top). groups ( $\mathrm{n}=6$ mice). Expression of Cav 3.1 was significantly up-regulated under 
908

909

910

911

913

914

915

916

917

918

919

920

921

922

923

924

925

926

927

928

between control $(n=16$ cells $)$ and chronic stress groups $(n=14$ cells $)$. Expression of

Cav 3.1 was significantly up-regulated under chronic stress conditions (unpaired Student's $t$-test, $P=0.0390)$. (d) Evoked burst firing trace of dmVMH neurons without and with T-VGCC antagonist (mibefradil, $10 \mu \mathrm{M}$ ), $10 \mathrm{pA}$ current injection was given in cosine waveform. (e) Effects of mibefradil on onset time and RMP of dmVMH neurons from wild-type $(n=15)$ and chronic stress groups $(n=13)$. Significant differences were observed in both onset time and RMP (paired Student's $t$-test, $P=0.0388$ and $P=$ 0.0395) in the stress group, whereas the control group demonstrated obvious changes in RMP but not onset time (paired Student's $t$-test, $P=0.0275$ and $P=0.3125$ ). (f)

Single-cell qRT-PCR analysis of Cav 3.1 expression among three neuronal subtypes in dmVMH. Upper: burst firing neurons $(n=11)$ showed higher Cav 3.1 expression than other two subtypes (unpaired Student's $t$-test, $P=0.0133$ compared with silent neurons $(\mathrm{n}=7), P=0.0139$ compared with tonic-firing neurons $(\mathrm{n}=12))$; bottom: Cav 3.1 expression in burst firing subtype showed significant differences between control and chronic-stress groups (unpaired Student's $t$-test, silent: control, $\mathrm{n}=3$, stress, $\mathrm{n}=3, P=$ 0.5574; tonic-firing: control, $\mathrm{n}=7$, stress, $\mathrm{n}=5, P=0.9339$; bursting: control, $\mathrm{n}=5$, stress, $\mathrm{n}=6, P=0.0165)$. (g) Effects of mibefradil on suprathreshold and subthreshold activity in dmVMH burst firing neurons in control $(\mathrm{n}=5)$ and chronic stress groups $(\mathrm{n}=$ 7). Mibefradil inhibited T-VGCC and caused a right frequency-current curve shift (two-way ANOVA, control, $P=0.2140, F(1,8)=1.854$; stress, $P=0.0077, F(1,12)=$ 10.22); lower, mibefradil application exerted no obvious influence on current-voltage 
929 curve of burst neurons (two-way ANOVA, control, $P=0.8188, F(1,10)=0.0573$;

930 stress, $P=0.4487, F(1,10)=0.6218)$. (h) Obvious differences were observed in

931 suprathreshold activity (two-way ANOVA, $P=0.0047, F(1,110)=20.53$ ), but not in

932 subthreshold (two-way ANOVA, $P=0.9913, F(1,33)=4.958$ ) membrane potential of

$933 \mathrm{dmVMH}$ burst firing neurons between control $(\mathrm{n}=5)$ and chronic stress groups $(\mathrm{n}=7)$

934 after application of mibefradil. Data are means \pm SEM. $* P<0.05, * * P<0.01$. 
a

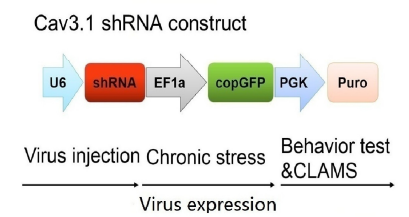

b
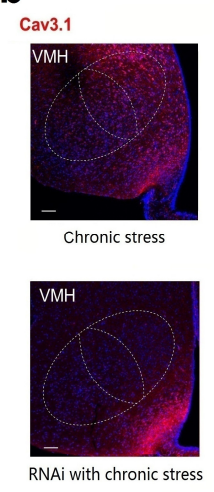

e

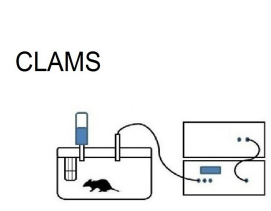

f

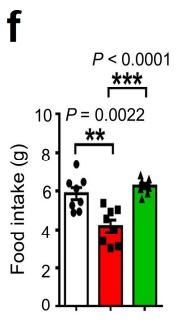

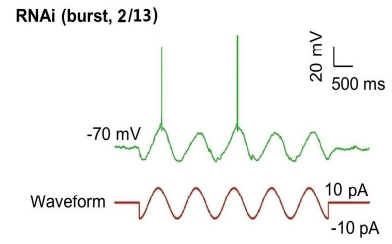

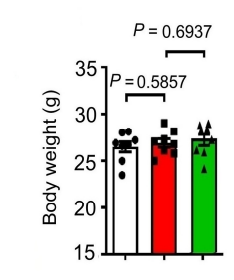

Stress (burst, 7/17)

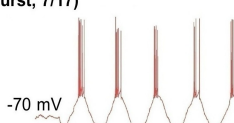

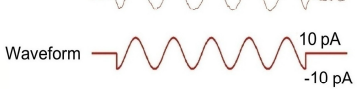

NAi (burst, 2/13)

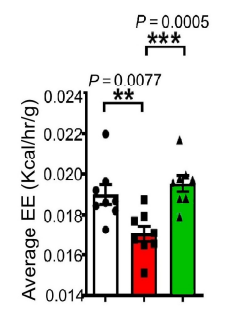

C

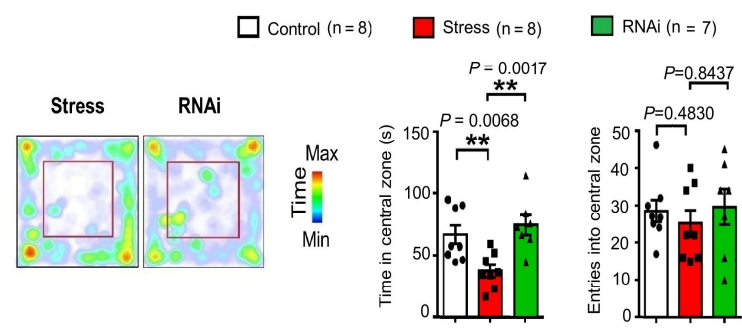

d
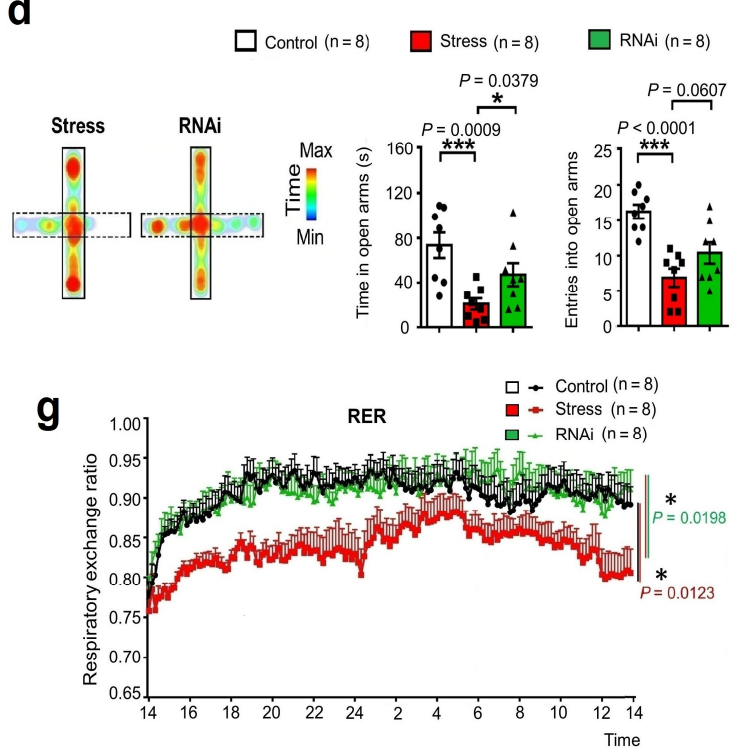

h

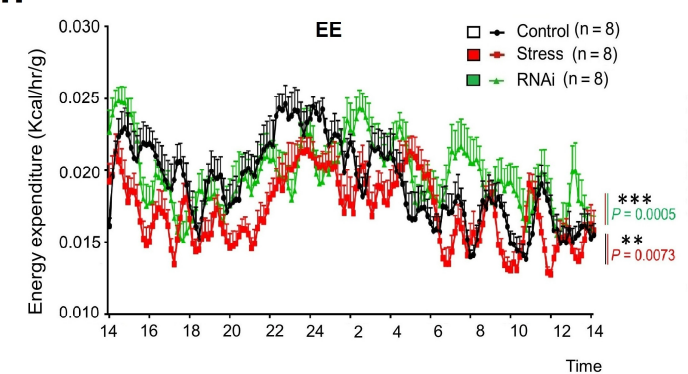

937 Fig. 5| Knockdown of Cav3.1 in dmVMH decreased burst firing, and rescued

939 (a) Schematic of Cav3.1 shRNA construct and injection of shRNA-expressing

940 lenti-viral vector into dmVMH to interfere with Cav 3.1 expression. (b) Representative

941 images of dmVMH Cav 3.1 immunostaining in chronic stress and RNAi (under chronic

942 stress) animals (left). Proportion of burst firing neurons decreased in RNAi group (2/13,

$94315.38 \%$ ) compared with stress group (7/17, 41.18\%) (right). Scale bar is $100 \mu \mathrm{m}$. (c) 
944 Time spent in central area and number of entries into central area of open field in

945 control, chronic stress, and RNAi groups. Residence time: control $(\mathrm{n}=8)$ versus

946 chronic stress group $(\mathrm{n}=8), P=0.0068$; chronic stress versus RNAi group $(\mathrm{n}=7), P=$

947 0.0017. Number of entries: control versus chronic stress group, $P=0.4830$; chronic

948 stress versus RNAi group, $P=0.8437$ (unpaired Student's $t$-test). (d) Time spent in

949 open arm and number of entries into open arm of elevated plus-maze in control, chronic

950 stress, and RNAi groups. Residence time: control versus chronic stress group, $P=$

9510.0009 ; chronic stress versus RNAi group, $P=0.0379$. Number of entries: control

952 versus chronic stress group, $P<0.0001$; chronic stress versus RNAi group, $P=0.0607$

953 (unpaired Student's $t$-test, 8 mice in each group). (e) No significant differences in

954 average body weights of control $(n=8)$, chronic stress $(n=8)$ and RNAi $(n=8)$ groups

955 were observed after four weeks of chronic stress (unpaired Student's $t$-test, control

956 versus stress group, $P=0.5857$; chronic stress versus RNAi group, $P=0.6937)$. (f)

957 Food intake of mice in control, chronic stress, and RNAi groups: control versus chronic

958 stress group, $P=0.0022$; chronic stress versus RNAi group, $P<0.0001$ (unpaired

959 Student's $t$-test, 8 mice in each group). Average RER of control, chronic stress, and

960 RNAi groups (unpaired Student's $t$-test: control versus chronic stress group, $P=0.0095$;

961 chronic stress versus RNAi group, $P=0.0032$ ). Average EE of control, chronic stress,

962 and RNAi groups (unpaired Student's $t$-test: control versus chronic stress group, $P=$

963 0.0077; chronic stress versus RNAi group, $P=0.0005)$. (g) 24-h RER curve of control,

964 chronic stress, and RNAi groups (two-way ANOVA: control versus chronic stress 
965 group, $P=0.0123, F(1,14)=8.244$; chronic stress versus RNAi group, $P=0.0198, F(1$,

966 13) $=7.055 ; 8$ mice in each group). (h) 24-h EE curve of control, chronic stress, and

967 RNAi groups (two-way ANOVA: control versus chronic stress group, $P=0.0005, F(1$,

$96814)=20.21$; chronic stress versus RNAi group, $P=0.0073, F(1,14)=9.851 ; 8$ mice in

969 each group). Data are means $\pm \mathrm{SEM}, * P<0.05, * * P<0.01, * * * P<0.001$. 
971 Table 1. Electrophysiological properties of three dmVMH neuronal subtypes

\begin{tabular}{lccc}
\hline & $\begin{array}{c}\text { Silent } \\
(\mathrm{n}=19)\end{array}$ & $\begin{array}{c}\text { Tonic-firing } \\
(\mathrm{n}=38)\end{array}$ & $\begin{array}{c}\text { Bursting } \\
(\mathrm{n}=28)\end{array}$ \\
\hline Input resistance $(\mathrm{M} \Omega)$ & $535.30 \pm 21.83$ & $661.72 \pm 19.84$ & $662.72 \pm 24.21$ \\
Onset Time (ms, 50 pA) & $37.17 \pm 2.53$ & $18.20 \pm 1.28$ & $25.84 \pm 1.87$ \\
RMP (mV) & $-65.27 \pm 1.08$ & $-57.50 \pm 0.92$ & $-63.39 \pm 1.07$ \\
$\begin{array}{l}\text { Overshoot by H-current } \\
\text { (mv, -100 pA) }\end{array}$ & $7.26 \pm 1.17$ & $10.71 \pm 0.94$ & $12.01 \pm 1.19$ \\
$\begin{array}{l}\text { Evoke firing rate (Hz, 100 } \\
\text { pA) }\end{array}$ & $17.53 \pm 0.60$ & $21.76 \pm 0.50$ & $22.00 \pm 0.73$ \\
AHP (mV) & $-12.38 \pm 0.53$ & $-15.2 \pm 0.50$ & $-14.26 \pm 0.65$ \\
Half width (ms) & $2.17 \pm 0.08$ & $2.00 \pm 0.04$ & $2.04 \pm 0.05$ \\
AP amplitude (mV) & $71.79 \pm 1.88$ & $73.29 \pm 1.25$ & $76.27 \quad \pm 1.28$ \\
\hline
\end{tabular}

972 Data are represented as mean \pm SEM. RMP: resting membrane potential; H-current:

973 hyperpolarization-activated current; AHP: after-hyperpolarization potential; AP: action

974 potential.

975 


\section{Supplemental Information}

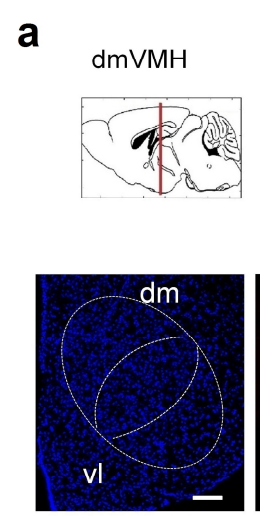

DAPI

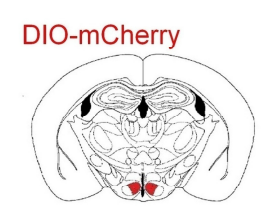

Sf1-Cre

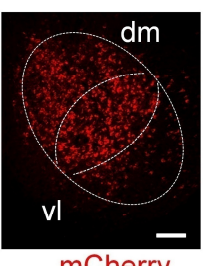

mCherry

d
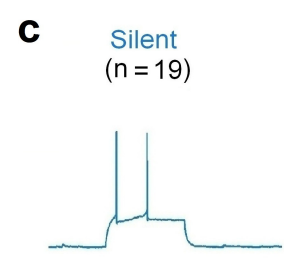

$-76 \mathrm{mV}$

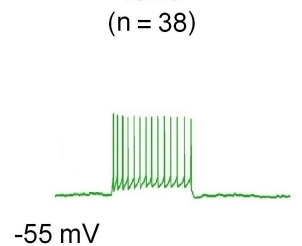

e

$$
\begin{gathered}
\text { Burst } \\
(n=28)
\end{gathered}
$$
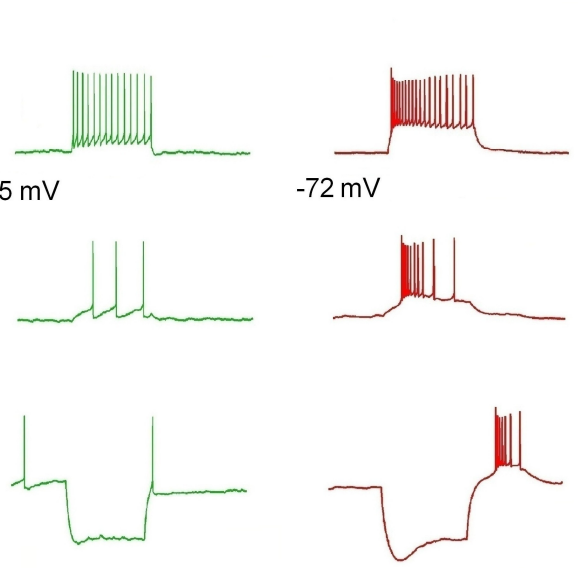

b
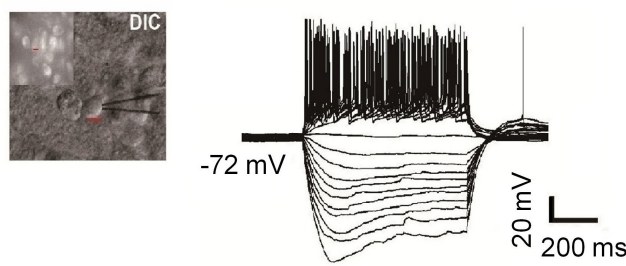

Waveform

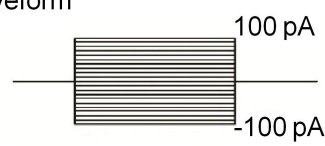

Waveform
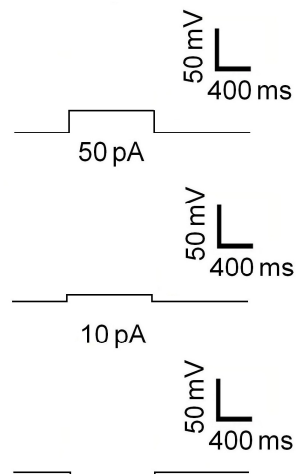

$-100 \mathrm{pA}$

\section{7}

978

979

980

981

982

983

984

Extended Data Fig. 1| Electrophysiological properties of dmVMH neurons

subtypes. Related to Fig. 2.

(a) Schematic of location of dmVMH in coronal section slice of mouse brain, mCherry was specifically expressed in SF-1 (specific dmVMH marker) neurons. Scale bar is 300 $\mu \mathrm{m}$. (b) Whole-cell recording trace from a dmVMH neuron, with current injection of $-100 \mathrm{pA}$ to $100 \mathrm{pA}$ and $10 \mathrm{pA} / \mathrm{step}$. (c-e), Representative traces of whole-cell recordings showing electrophysiological properties of silent $(n=19)$, tonic-firing $(n=$ 38), and bursting $(\mathrm{n}=28)$ dmVMH neuronal subtypes. Three subtypes exhibit different 
bioRxiv preprint doi: https://doi.org/10.1101/2020.12.20.423695; this version posted December 22, 2020. The copyright holder for this preprint (which was not certified by peer review) is the author/funder, who has granted bioRxiv a license to display the preprint in perpetuity. It is made available under aCC-BY-NC-ND 4.0 International license.

986 electrophysiological activity at 50,10, and -100 pA current injection.

987 
a
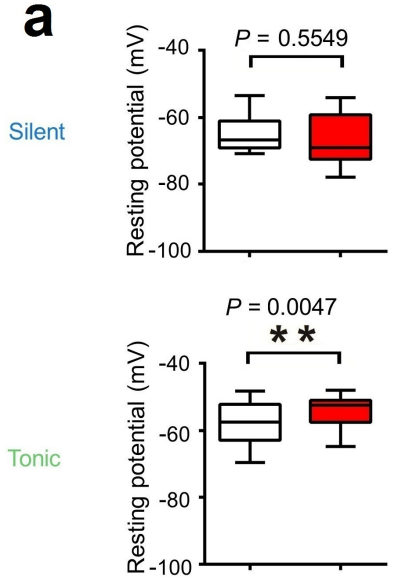

b
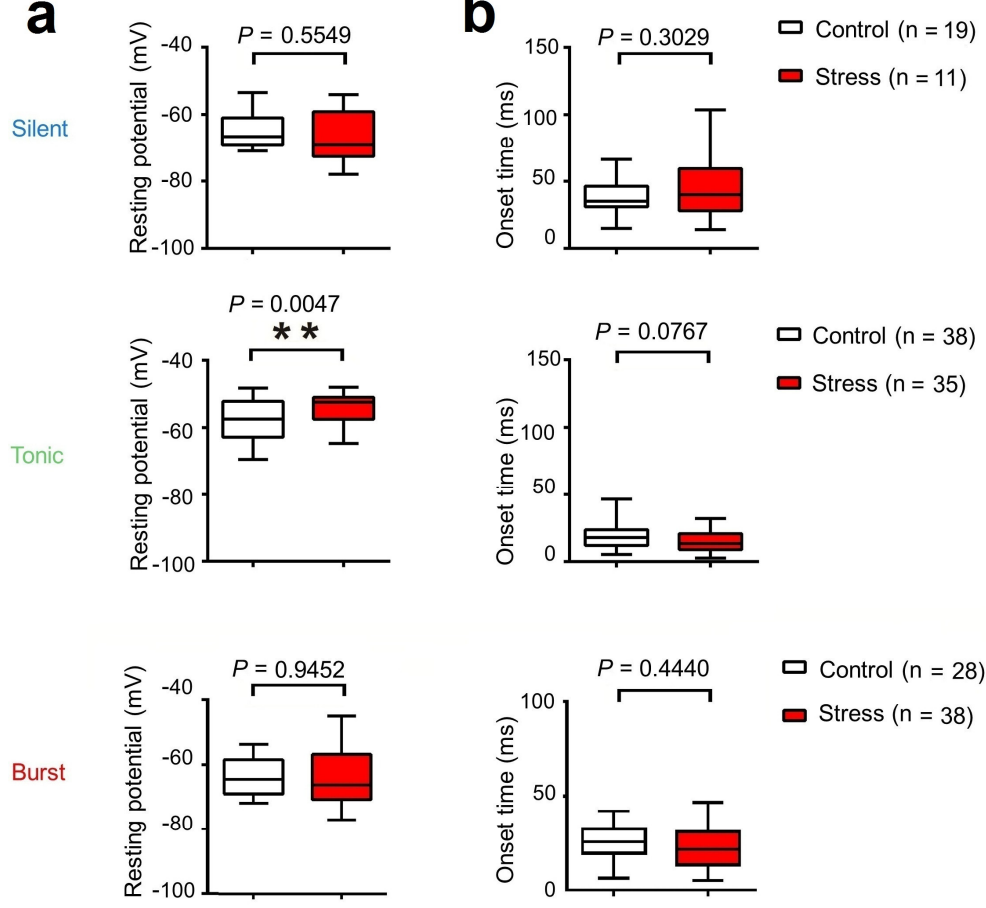

C

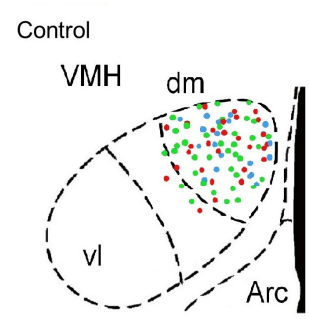

Stress

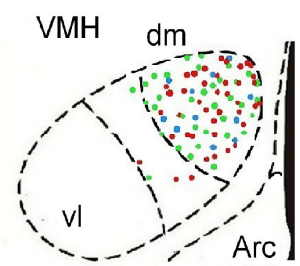

口 Control $(n=28)$

$\square$ Stress $(n=38)$

Extended Data Fig. 2| Electrophysiological comparison of three dmVMH

990 neuronal subtypes in control and chronic stress groups. Related to Fig. 2.

991 (a) Resting membrane potential (RMP) of three neuronal subtypes in control and

992 stressed mice. Top: silent neurons in control $(\mathrm{n}=19)$ and chronically stress mice $(\mathrm{n}=$

$99313), P=0.5549$; Middle: tonic-firing neurons in control $(\mathrm{n}=38)$ and chronically

994 stress mice $(\mathrm{n}=33), P=0.0047$; Bottom: bursting neurons in control $(\mathrm{n}=29)$ and

995 chronically stress mice $(\mathrm{n}=38), P=0.9452$. (b) Onset time of three neuronal

996 subtypes in control and stressed mice. Top: silent neurons in control $(\mathrm{n}=19)$ and

997 chronically stress mice $(\mathrm{n}=13), P=0.3029$; Middle: tonic-firing neurons in control 
$998(\mathrm{n}=39)$ and chronically stress mice $(\mathrm{n}=33), P=0.0767$; Bottom: bursting neurons in

999 control $(\mathrm{n}=27)$ and chronically stress mice $(\mathrm{n}=38), P=0.7704$ (unpaired Student's

$1000 t$-test). (c) Location of each recorded neuron in dmVMH of control and stressed group,

1001 no region specificity was observed among three subtypes. Data are means $\pm \mathrm{SEM}$; $P$

$1002<0.05, * * P<0.01$. The box plotted at the median extending from the $25-75$ th

1003 percentile, and the whisker represents Min to Max distribution. 
a

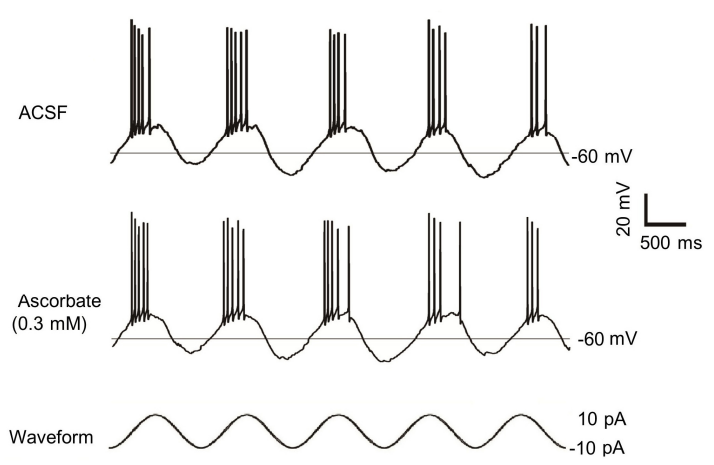

b

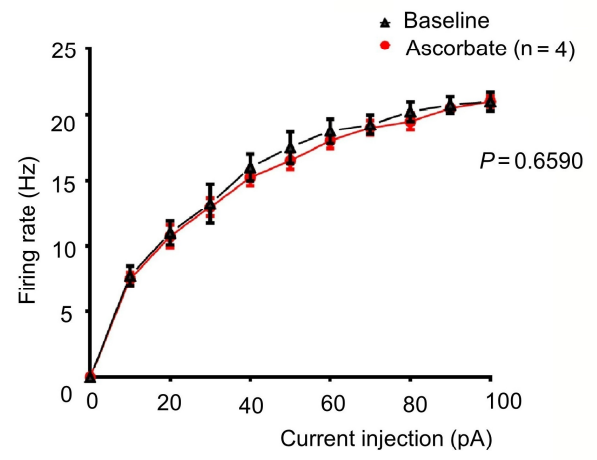

(a) Ascorbate (antagonist of Cav3.2) did not block burst firing of dmVMH neurons ( $\mathrm{n}=$

4) induced by 10-pA current injection in cosine waveform.

(b) Ascorbate imposed no obvious influence on burst firing of dmVMH neurons, with 


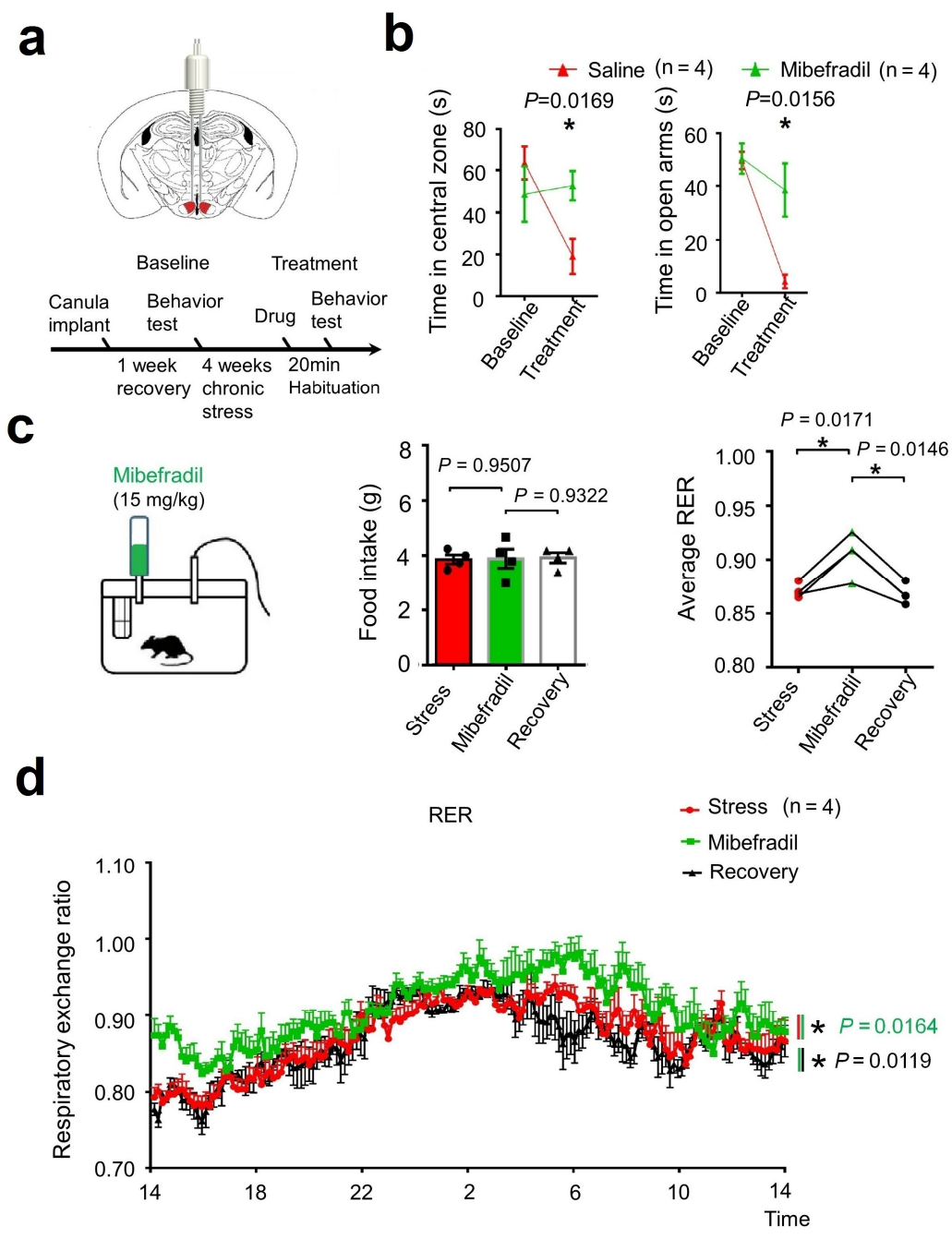

1017 (a) Schematic of microinjection of mibefradil in dmVMH through cannula. (b)

1018 Behavioral test before and after drug delivery; left, residence time in central area of 1019 open field of saline group decreased compared with mibefradil group $(n=4$ in each 1020 group, $P=0.0169$, unpaired Student's $t$-test); right, residence time in open arm of saline 1021 group decreased compared with mibefradil group $(\mathrm{n}=4$ in each group, $P=0.0156$, unpaired Student's $t$-test). (c-d) Oral administration of mibefradil exerted no obvious change on food intake ( $\mathrm{n}=4$ in each group, $P=0.9507$, unpaired Student's $t$-test) but 
bioRxiv preprint doi: https://doi.org/10.1101/2020.12.20.423695; this version posted December 22, 2020. The copyright holder for this preprint (which was not certified by peer review) is the author/funder, who has granted bioRxiv a license to display the preprint in perpetuity. It is made available under aCC-BY-NC-ND 4.0 International license.

1024 increased average RER in stressed mice $(P=0.0171$, unpaired Student's $t$-test). Data

1025 are means $\pm \mathrm{SEM} ; * P<0.05$.

1026

1027 
a
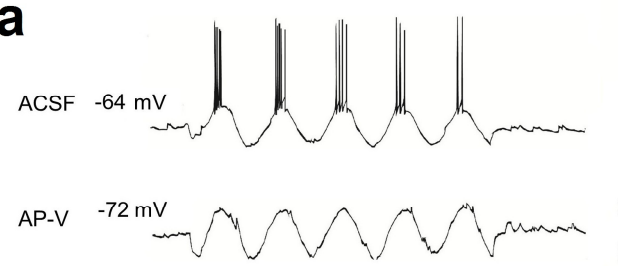

Waveform

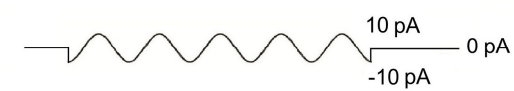

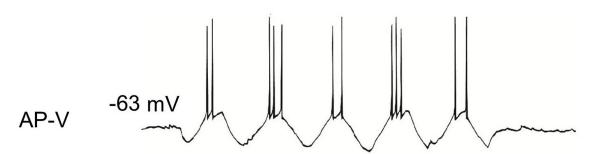

Waveform

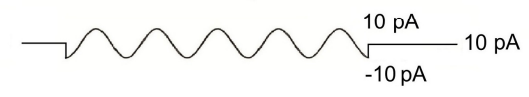

b
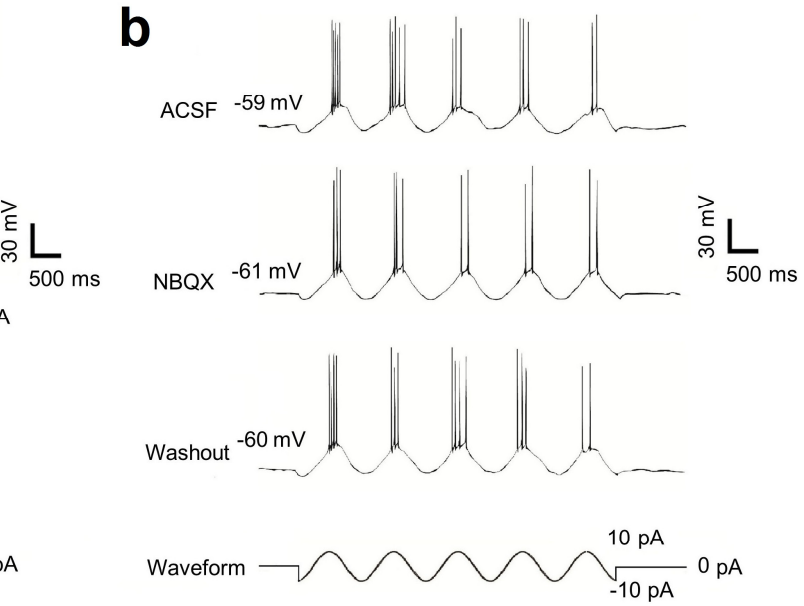

(a) Application of APV blocked generation of burst firing induced by cosine waveform did not abolish generation of burst firing $(n=3)$. 

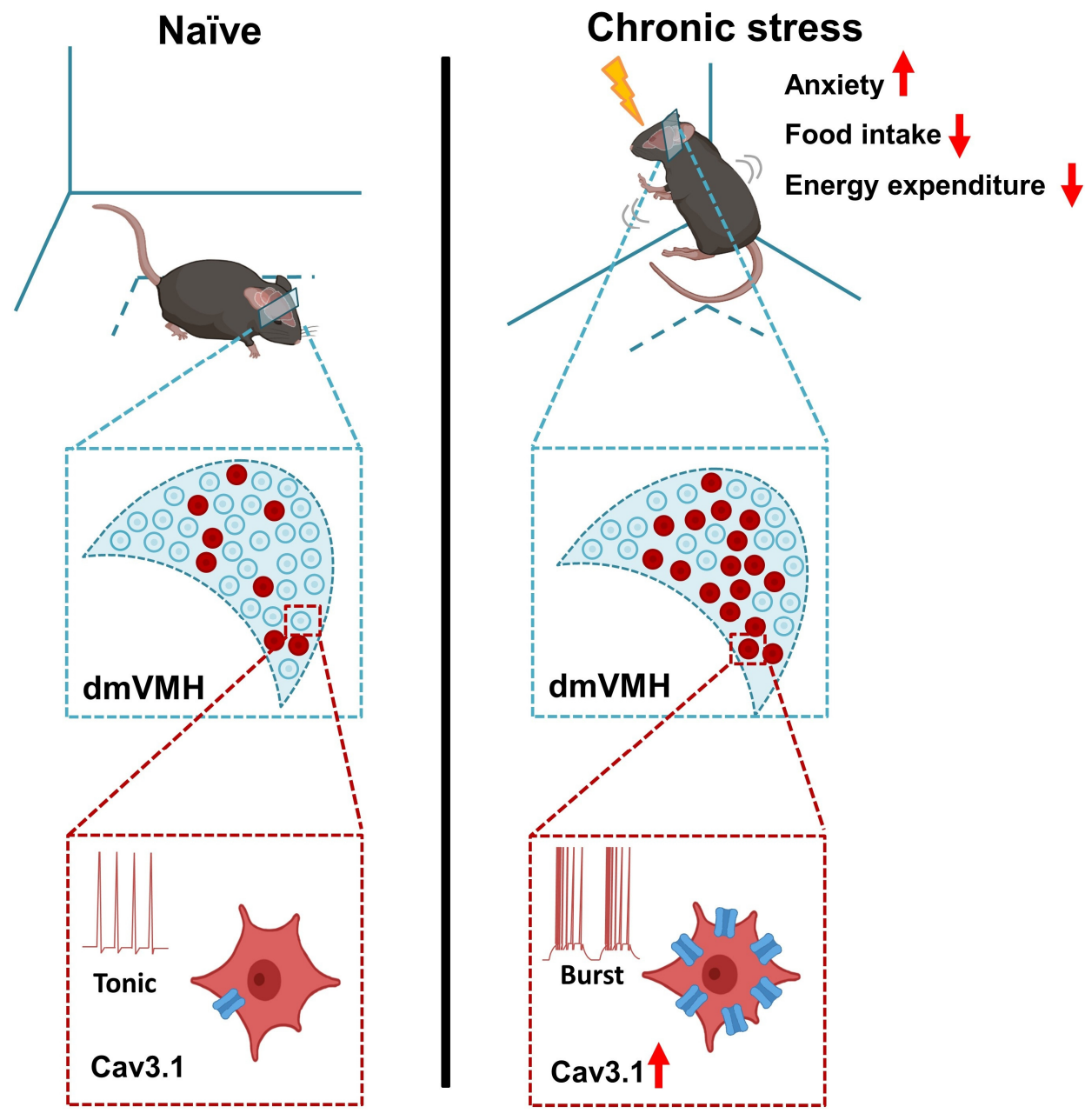

Extended Data Fig. 6| Schematic representation of the enhancement of burst elevated expression of Cav3.1. 